Published in "Biochimica et Biophysica Acta (BBA) -

General Subjects doi: 10.1016/j.bbagen.2014.04.008, 2014"

which should be cited to refer to this work.

\title{
Structure and mechanism of ATP-dependent phospholipid transporters ${ }^{\text {tr }}$
}

\author{
Rosa L. López-Marqués a,*, Lisbeth Rosager Poulsen ${ }^{\mathrm{a}}$, Aurélien Bailly ${ }^{\mathrm{b}}$, Markus Geisler ${ }^{\mathrm{c}}$, \\ Thomas Günther Pomorski ${ }^{\mathrm{a}}$, Michael G. Palmgren ${ }^{\mathrm{a}, * *}$ \\ a Centre for Membrane Pumps in Cells and Disease - PUMPkin, Department of Plant and Environmental Sciences, University of Copenhagen, Thorvaldsensvej 40, DK-1871 Frederiksberg C, Denmark \\ ${ }^{\mathrm{b}}$ Institute of Plant Biology, University of Zürich, Zollikerstrasse 107, CH-8008 Zürich, Switzerland \\ c Department of Biology, Plant Biology, University of Fribourg, Chemin du Musée 10, CH-1700 Fribourg, Switzerland
}

Background: ATP-binding cassette (ABC) transporters and P4-ATPases are two large and seemingly unrelated families of primary active pumps involved in moving phospholipids from one leaflet of a biological membrane to the other.

Scope of review: This review aims to identify common mechanistic features in the way phospholipid flipping is carried out by two evolutionarily unrelated families of transporters.

Major conclusions: Both protein families hydrolyze ATP, although they employ different mechanisms to use it, and have a comparable size with twelve transmembrane segments in the functional unit. Further, despite differences in overall architecture, both appear to operate by an alternating access mechanism and during transport they might allow access of phospholipids to the internal part of the transmembrane domain. The latter feature is obvious for ABC transporters, but phospholipids and other hydrophobic molecules have also been found embedded in P-type ATPase crystal structures. Taken together, in two diverse groups of pumps, nature appears to have evolved quite similar ways of flipping phospholipids.

General significance: Our understanding of the structural basis for phospholipid flipping is still limited but it seems plausible that a general mechanism for phospholipid flipping exists in nature. This article is part of a Special Issue entitled Structural biochemistry and biophysics of membrane proteins.

\section{Background}

Biological membranes are the basis for highly defined and separated functional units. The cell membrane, or plasma membrane, defines the external boundary of every cell separating the cytoplasm from the surrounding environment. Eukaryotic cells contain in addition numerous subcellular membranes that divide the cytoplasm into multiple organelles, thereby allowing different functions to occur efficiently and simultaneously in different parts of the cell. Almost all biological membranes are organized as bilayers consisting of two leaflets structurally formed by phospholipids. Depending on their (sub)cellular location, they might also contain other types of phospholipids, including glycolipids and sterols. A remarkable feature of many biological membranes is that their phospholipids are asymmetrically distributed across the lipid bilayer, a phenomenon known as transbilayer lipid asymmetry. A prominent example is the plasma membrane of animal cells where

This article is part of a Special Issue entitled Structural biochemistry and biophysics of membrane proteins.

* Corresponding author. Tel.: +4535332602.

** Corresponding author. Tel.: +4535332592.

E-mail addresses: rlo@plen.ku.dk (R.L. López-Marqués), palmgren@plen.ku.dk (M.G. Palmgren) the phospholipids phosphatidylcholine (PC) and sphingomyelin (SM) are concentrated in the exoplasmic leaflet while phosphatidylserine (PS) and phosphatidylethanolamine (PE) are restricted to the cytosolic leaflet [1]. Transbilayer lipid asymmetry is essential for several vital cellular functions, including regulation of membrane protein activity, signaling, and vesicle formation in the secretory and endocytic pathways [2-5]. In animals, loss of transbilayer lipid asymmetry has been related to processes like blood coagulation [6], macrophage recognition [7] and apoptosis [8]. Establishing and maintaining the asymmetry is thus crucial for the cells, and a number of proteins have evolved to fulfill a role as cross-bilayer phospholipid transporters.

Transbilayer lipid asymmetry is largely controlled by a diverse group of membrane proteins that catalyze the movement of phospholipids across membranes. Lipid translocators can be classified into two categories: (i) energy-independent transporters such as scramblases that randomize the distribution of lipids across the bilayer and (ii) ATP-driven, vectorial transporters that actively translocate specific lipids from one leaflet to the other. The latter class of transporters includes ATPdependent flippases and floppases, which catalyze inward phospholipid movement to and outward phospholipid movement from the cytoplasmic leaflet of cellular membranes, respectively. Current genetic and biochemical evidence indicates that these proteins are primarily members of the ATP-binding cassette $(A B C)$ and P-type family of transporters 
(Tables 1 and 2). However, measuring phospholipid translocation is not a trivial task, as the transporters are trapped in an environment (cellular membranes) formed by their own substrate (lipids). Therefore, in intact cells or organisms, assignment of phospholipid translocating activity is in most cases based on the use of fluorescent phospholipid analogs; only few studies have attempted to measure transport of natural phospholipids (Tables 1 and 2). Phospholipid transport observed in vivo need not be directly linked to the activity of ABC transporters or P4ATPases, and might represent indirect effects. Only recently, advances in purification and reconstitution techniques have allowed demonstrating the capacity of some ABC transporters and P-type ATPases to directly translocate fluorescent phospholipid analogs, providing the best evidence so far that these transporters indeed have phospholipids as a substrate.

\section{Scope of review}

Several excellent reviews have surveyed the physiological relevance of phospholipid transporters recently [3,5,9]. Here, we will focus on recent advancements in the determination of the structure and mechanism of putative ATP-dependent phospholipid transporters.

\section{ATP-dependent phospholipid transporters}

Cellular, biochemical and recent reconstitution studies demonstrate that various ABC transporters and P-type ATPases couple ATP hydrolysis to translocation of specific phospholipids from one leaflet to the other and thereby help generate membrane lipid asymmetry (Tables 1 and 2).
An important question arises when comparing the two classes of phospholipid flipping pumps: are their transport mechanisms the same or has nature evolved different ways of translocating phospholipids across the bilayer? In the next sections, we will review the main features of ABC transporters and P-type ATPases implicated in phospholipid transport and compare their putative transport mechanism(s).

\section{1. $A B C$ transporter family}

The $A B C$ transporter family is found in organisms throughout evolution and constitutes one of the largest superfamilies of integral membrane transporters. The family includes a wide range of proteins that share common structural features and transport a variety of organic and inorganic substrates, including phospholipids (Table 1). Several of these transporters are mutated in human disorders related to phospholipid transport and metabolism, including Tangier disease [10], Stargardt disease [11] and progressive familial intrahepatic cholestasis [12]. The functional transport unit comprises two nucleotide-binding domains (NBD), typical of ABC transporters, and two transmembrane domains (TMDs), each containing five to ten membrane-spanning regions (Fig. 1A). They can occur as one complete transporter, two half-transporters, or four polypeptides.

\subsubsection{General structural features of $A B C$ transporters}

The NBDs of all $A B C$ transporters contain highly conserved amino acid motifs. Each NBD contains two ATP-binding motifs: the Walker A motif (GXXGXGK(S/T)) and the Walker B motif (hhhhDE, where $h$ is a

Table 1

Evidence for $\mathrm{ABC}$ transporter-catalyzed phosholipid transport.

\begin{tabular}{|c|c|c|c|c|}
\hline Transporter & Lipid $^{1}$ & $\begin{array}{l}\text { Movement, } \\
\text { membrane }^{2}\end{array}$ & Approach & Reference \\
\hline \multicolumn{5}{|l|}{ Bacteria } \\
\hline MsbA & Lipid A, PLs? & Out, PM & $\begin{array}{l}\text { Intact cells; ATPase stimulation; } \\
\text { Reconstitution }\end{array}$ & [72,137-139] \\
\hline PglK & Lipid-linked oligosaccharides & Out, PM & Intact cells & {$[140]$} \\
\hline LmrA & PE, Lipid A & Out, PM & $\begin{array}{l}\text { Reconstitution; } \\
\text { E. coli complementation; } \\
\text { ATPase stimulation }\end{array}$ & {$[141,142]$} \\
\hline \multicolumn{5}{|l|}{ Fungi } \\
\hline Pdr5p, Yor1p & PE, & Out, PM & Intact cells & {$[143,144]$} \\
\hline Ybt1p & $\mathrm{PC}$ & Out, vacuolar & $\begin{array}{l}\text { Intact cells; } \\
\text { Isolated vacuoles }\end{array}$ & {$[145]$} \\
\hline Cdr1p & PE,PC,PS & Out, PM & Intact cells; Reconstitution & {$[63,146,147]$} \\
\hline $\mathrm{Cdr} 2 \mathrm{p}$ & PE,PC,PS & Out, PM & Intact cells & [63] \\
\hline Cdr3p & PE,PC,PS & In, PM & Intact cells & [63] \\
\hline \multicolumn{5}{|l|}{ Leishmania donovani } \\
\hline LABCA 1 & PLs & Out, PM & Intact cells & [148] \\
\hline LABCA2 & PLs & Out, PM & Intact cells & [149] \\
\hline LABCB4 & PC & Out, PM & Intact cells & [150] \\
\hline LABCG2 & PS & Out, PM & Intact cells & [151] \\
\hline LABCG4 & PC & Out, PM & Intact cells; yeast vesicle assay & [152] \\
\hline LABCG6 & PLs & Out, PM & Intact cells & [153] \\
\hline \multicolumn{5}{|l|}{ Mammals } \\
\hline ABCA1 & PC, PS, SM & Out, PM & $\begin{array}{l}\text { Intact cells; } \\
\text { reconstitution }\end{array}$ & {$[65,91]$} \\
\hline ABCA3 & PC, SM & Out, lysosomal vesicles & Expression in HEK-293 and A549 & {$[154-156]$} \\
\hline ABCA4 & PE, N-retinylidene-PE & In, Disk & Reconstitution & {$[64,65,157]$} \\
\hline ABCA7 & PS, (PC, SM) & Out, PM & $\begin{array}{l}\text { Intact cells; } \\
\text { reconstitution }\end{array}$ & {$[65,158-160]$} \\
\hline ABCB1 (MDR1) & PLs, SLs, PAF & Out, PM (apical) & Intact cells; yeast vesicle assay; reconstitution & {$[70,71,89,90,161-164]$} \\
\hline ABCB4 (MDR3,Mdr2) & PC & Out, canalicular & Intact cells; yeast vesicle assay & {$[89,165-167]$} \\
\hline ABCC1 (MRP1) & PLs & Out, PM (basolateral) & Intact cells; reconstitution & {$[168-171]$} \\
\hline
\end{tabular}

${ }^{1}$ PC, phosphatidylcholine; PE, phosphatidylethanolamine; PS, phosphatidylserine. PLs, phospholipids; SLs, sphingolipids; PAF, platelet-activating factor (1-Hexadecyl-2-acetyl-snglycero-3-phosphocholine); SM, sphingomyelin. Substrate specificities are mostly demonstrated by the use of fluorescent lipid probes. Evidences for translocation of natural lipids are indicated in bold.

2 PM, plasma membrane; Disk, photoreceptor disk membranes. 
Table 2

Evidence for P4-ATPase-catalyzed phosholipid transport.

\begin{tabular}{|c|c|c|c|c|}
\hline Transporter & Lipid $^{1}$ & $\begin{array}{l}\text { Movement, } \\
\text { membrane }^{2}\end{array}$ & Approach & Reference \\
\hline \multicolumn{5}{|c|}{ Saccharomyces cerevisiae } \\
\hline Dnf1p & PC, PE, (PS), LPC, LPE, LPS & In, PM & Intact cells & {$[126,143,172,173]$} \\
\hline Dnf2p & PC, PE, (PS), LPC, LPE & In, PM & Intact cells & {$[143,172-174]$} \\
\hline Dnf3p & PC, PE & In, Golgi & Purified vesicles & {$[175]$} \\
\hline Drs2p & PS, PE & In, Golgi & Purified vesicles; Reconstitution & {$[174,176]$} \\
\hline \multicolumn{5}{|c|}{ Leishmania donovani } \\
\hline LdMT & PC, PE, (PS) & In, PM & Intact cells & {$[177,178]$} \\
\hline \multicolumn{5}{|c|}{ Caenorhabditis elegans } \\
\hline TAT-1 & PS & In, PM & Intact cells & {$[179,180]$} \\
\hline TAT-5 & PE & In, PM & Intact cells & {$[181]$} \\
\hline \multicolumn{5}{|c|}{ Arabidopsis thaliana } \\
\hline ALA1 & $(\mathrm{PS})$ & In, PM & Yeast complementation & {$[104,182]$} \\
\hline ALA2 & PS & In, Prevacuolar compartment & Yeast complementation & [114] \\
\hline ALA3 & PS, PE, PC & In, Golgi & Yeast complementation & {$[113,114]$} \\
\hline \multicolumn{5}{|l|}{ Mammals } \\
\hline ATP8A1 & PS, (PE) & In, Golgi & $\begin{array}{l}\text { ATPase stimulation; } \\
\text { Yeast vesicle assay }\end{array}$ & [183-186] \\
\hline ATP8A2 & PS, PE & Golgi, Disks & Reconstitution & {$[124,187,188]$} \\
\hline ATP8B1 & PS, (PE) & PM (apical) & Intact cells & {$[115,189,190]$} \\
\hline ATP8B3 & PS & PM & Intact cells & [191] \\
\hline ATP11C & PS & PM & Intact cells & [192] \\
\hline
\end{tabular}

1 PC, phosphatidylcholine; PE, phosphatidylethanolamine; PS, phosphatidylserine; LPC, lyso-phosphatidylcholine; LPE, lyso-phosphatidylethanolamine; LPS, lyso-phosphatidylserine PLs, phospholipids; SLs, sphingolipids; PAF, platelet-activating factor (1-Hexadecyl-2-acetyl-sn-glycero-3-phosphocholine). Substrate specificities are mostly demonstrated by the use of fluorescent lipid probes. Evidences for translocation of natural lipids are indicated in bold.

2 PM, plasma membrane; AM, apical membrane; Disk, photoreceptor disk membranes.

hydrophobic amino acid) [13,14]. The Walker A motif establishes extensive interactions with the phosphate group of an ATP molecule [15,16]. The Walker B motif contains an aspartate involved in the coordination of water and $\mathrm{Mg}^{2+}$ at the catalytic site and a catalytic glutamate required for ATP hydrolysis [16-19]. A similar role in coordination has a conserved glutamine at the Q-loop located between the Walker sequences [16,19-21]. A conserved aromatic amino acid residue in the A-loop upstream of the Walker A motif is involved in interacting with the $\pi$-stack of the adenosine ring of ATP [22-24]. Downstream of Walker B are the D-loop and the $\mathrm{H}$ (or switch)-loop that contain a conserved aspartate and a conserved histidine, respectively, involved in the coordination of the $\gamma$-phosphate either through interaction with a water molecule (D-loop) [17,19,20,25,26] or through direct hydrogen bonding (H-loop) [19,27]. Finally, the ABC signature is a helical subdomain located in between the Walker sequences of each NBD containing the amino acid sequence LSGGQ also involved in ATP binding [28-33].

When the transporter binds ATP, the two NBDs arrange in a head-totail manner, generating the so-called "closed-dimer" conformation, in which two nucleotide molecules are sandwiched in the interface between the two NBDs and interact with residues from the Walker $A$, Walker B, H- and Q-loops of one NBD and the D-loop and ABC signature of the other $[15,17,34]$. Due to this arrangement some $A B C$ transporters show an allosteric behavior for hydrolysis of the two ATP molecules, presumably controlled by the D-loop $[26,33,35]$.

$A B C$ transporters present three different types of structural folding, depending on the organization of their transmembrane segments [36,37]. Thus, it is possible to distinguish between an "exporter" fold adopted by proteins involved in the transport of substrates away from the cytoplasm, and two distinct "importer" folds, typical of proteins that transport substrates toward the cytoplasm.

The typical ABC "exporter" fold contains a total of 12 transmembrane spanning $\alpha$-helices that form the substrate binding site and provide the pathway for the movement of substances across the membrane [36] (Fig. 1A). TMDs from different transporters present low levels of homology, probably reflecting the wide variety of substrates transported by this protein family. The 12 membrane-spanning segments arrange into two bundles of six with a large cavity in between that can either be open-inwards (to the cytosol) or open-outwards (to the extracellular/ luminal side) (Fig. 1B). In the open-inwards conformation, each bundle is commonly formed by interaction between transmembrane segment 4 (TM4) and TM5 of one half transporter or half monomer and TM3 and TM6 of the other half. In this conformation, a 2-fold pseudo-symmetry can be observed for TM1-3 with respect to TM4-6 for each individual TMD [38-40]. During catalysis, the TMDs suffer extensive structural rearrangement establishing a new interaction surface that changes the orientation of the exposed cavity in the membrane [38,39,41-43]. This reorganization results in the "open-outwards" conformation in which TM1 and TM2 in one monomer now interact with TM3 and TM6 of the other [44]. In the process, a high affinity binding site for the substrate in the "open-inwards" conformation changes into a low affinity binding site in the "open-outwards" conformation $[45,46]$. To date, only the bacterial $\mathrm{ABC}$ transporter MsbA has been crystalized in both the inward and outward facing conformations presumably adopted by the "exporters" during the catalytic cycle (Table 3) [39]. However, the similarities between the different structures suggest that the alternating-opening conformations also exist for other transporters.

In ABC "exporters", those TMDs that are not directly connected to NBDs are extended by $\alpha$-helices that stretch out into the cytoplasm between 25 and $40 \AA$ away from the surface of the lipid bilayer $[40,47,48]$ (Fig. 1E and F). Such extended $\alpha$-helices are not found in the "importer" fold, suggesting that they might be directly related to the exporter type mechanism and, in some $\mathrm{ABC}$ exporters, they have been linked to recognition of a soluble transported substrate [49-51]. All together, the cytosolic protrusions of TMD helices generate four intracellular loops (IL1-IL4) that contain the so-called coupling helices. These run parallel to the plane of the membrane and interact with the NBDs [36,52,53]. Due to the twisted arrangement of the transmembrane spanning segments, IL2 and IL4, each located in a different half transporter or half monomer, interact exclusively with the trans-NBD (in the opposite half), while IL1 and IL3 only interact with the cis-NBD. Besides, TM6 finishes in a cytoplasmic $\alpha$-helix extension located just upstream of 
A

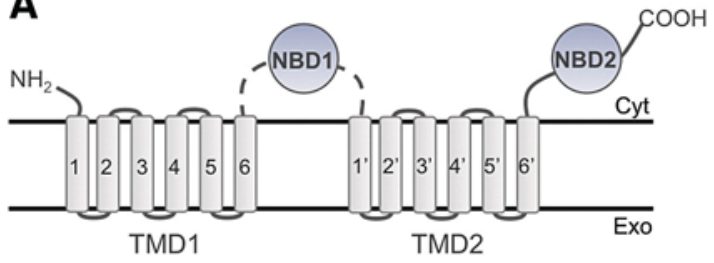

C

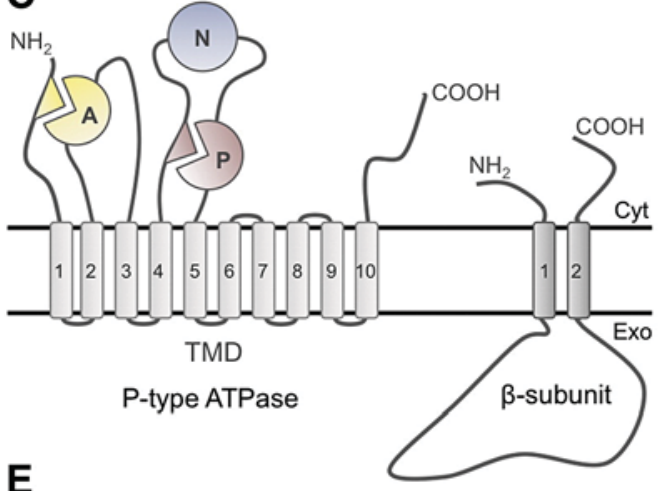

E

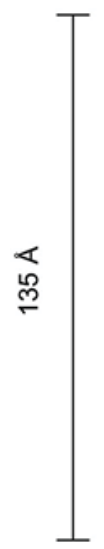

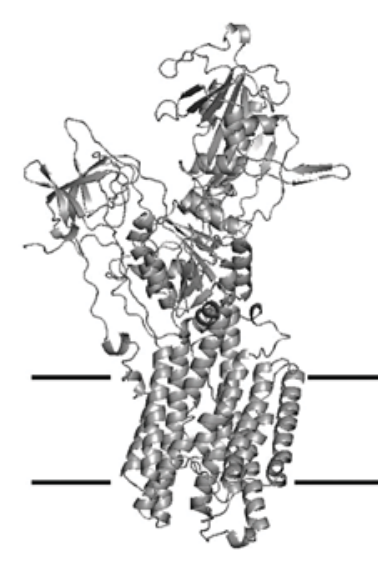

Dnf1p
B

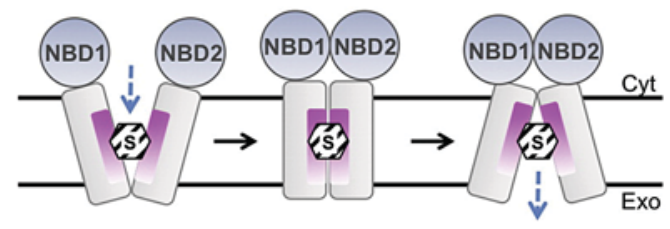

D

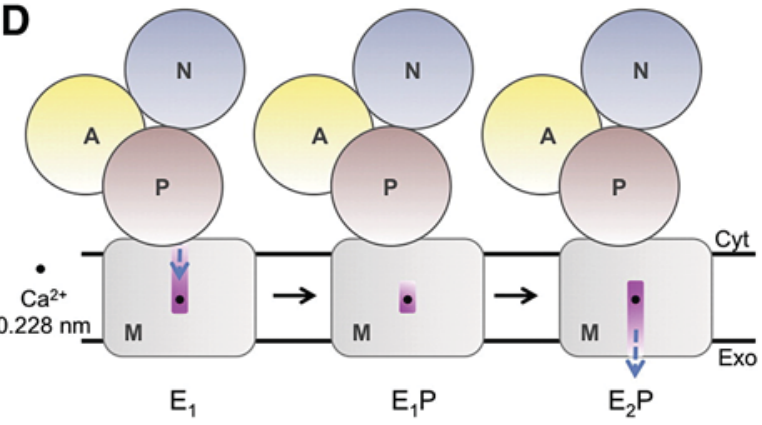

F

Fig. 1. General structure of $A B C$ transporters and P-type ATPases. (A) Secondary structure of ABC transporters. The ABC transporter exists both as a monomer full transporter and as a dimer consisting of two half transporters. In either case, this type of transporter contains two transmembrane domains (TMD1 and 2), each harboring 6 transmembrane helices named 1 to 6 in TMD1 and 1' to 6' in TMD2. Furthermore, two nucleotide binding domains (NBD1 and NBD2) are present on the cytosolic side of the membrane. NBD1 is either situated at the C-terminal end of one half transporter or is connecting TMD1 and TMD2 in the full transporter; alternatively the domain architecture can have a reverse topology, i.e. NBD1-TMD1-NBD2-TMD2. (B) Model of substrate translocation carried out by ABC exporters. First, the substrate enters the cavity between the transmembrane domains, which are facing the cytosol. At this stage the transporter has high affinity for the substrate. Second, the substrate is occluded in the ABC transporter. Third, through conformational changes a cavity is now opened toward the exocytic side of the membrane the substrate specificity decreases and the substrate is being released. (C) Secondary structure of P-type ATPases. These transporters consist of one transmembrane domain (TMD) with eight to twelve transmembrane helices labeled 1 to 12. Both the $\mathrm{N}$ - and $\mathrm{C}$-terminal ends are facing the cytosolic side of the membrane. The cytosolic domain of the transporter is divided into three major domains; The Actuator domain (A), the Nucleotide binding domain (N) and the phosphorylation domain (P). The $\mathrm{P}_{4}$-ATPases in addition to the $\alpha$ subunit consist of a $\beta$-subunit with two transmembrane spans and a large exoplasmic loop. (D) Model of ion transport through P-type ATPases. During the catalytical cycle the P-type ATPases undergoes large conformational changes during which a half channel is firstly opened toward the cytosolic side of the membrane. In this conformation the transporter has high affinity for the transported substrate. The enzyme becomes phosphorylated and undergoes a conformational change leading to the occlusion of the substrate followed by a closure of the half channel. An addition change in the conformation of the enzyme leads to the generation of an open half channel toward the exoplasmic side of the membrane in this conformation the substrate specificity decreases and the substrate is released. (E) Structural comparison between ABC transporters and P-type ATPases. Left, model of the yeast P4-ATPase Dnf1p [118]; Right, Salmonella typhimurium ABC transporter MsbA (PDB code 3B60). (F) Schematic model of the position of intracellular loops (ILs) in an ABC exporter. Light and dark gray colors represent the two independent halves of the transporter. ILs are designated by numbers (1-4). IL1 and IL2, corresponding to the first half transporter, interact with the cis-NBD and the trans-NBD, respectively. The same is the case for IL3 and IL4, corresponding to the second half transporter.

the NBD domain of each half monomer [39,40,52]. These interactions may allow for intramolecular communication between the NBDs and the TMD within the $A B C$ transporter.

ABC "importers" present two distinct types of fold, Type I and Type II. Type I transporters mediate uptake of relatively small substrates (sugars, ions and amino acids) and are structurally represented by the bacterial molybdate transporter ModBC $[54,55]$. They typically contain 12 transmembrane segments, arranged into two core bundles of five helices in each half transporter or half monomer, with an $\mathrm{N}$-terminal extra helix that wraps around the TMD of the other half transporter or half monomer [36]. In some cases, this extra helix is lacking, such as for the E. coli methionine importer MetNI [56], or additional regulatory helices might be present, as is the case for the bacterial maltose transporter MalFGK 2 [33,57]. Further biochemical and structural analyses are required to understand the consequences of this phenomenon for substrate movement.

Type II ABC "importers", involved in the transport of much bigger molecules, such as heme, metal chelates or vitamin B12, generally 
Table 3

Available crystal structures for $\mathrm{ABC}$ transporters of the exporter fold.

\begin{tabular}{|c|c|c|c|}
\hline Protein & Conformation & PDB entry* & Reference \\
\hline \multirow[t]{2}{*}{ Sav1866 } & Outward facing & $20 \mathrm{NJ}(3.40)$ & {$[45]$} \\
\hline & Inward facing & 2HYD (3.00) & [41] \\
\hline \multirow[t]{2}{*}{ MsbA } & Outward facing & $3 \mathrm{~B} 60$ (3.70), 3B5Y (4.50), 3B5Z (5.20) & [39] \\
\hline & Inward facing & 3B5W (5.30), 3B5X (5.50) & [39] \\
\hline TM287/288 & Inward facing & 3QF4 (3.90) & [48] \\
\hline ABCB10 & Inward facing & 3ZDQ (2.85), 4AYW (3.30), 4AYX (2.90), 4AYT (2.85) & {$[40]$} \\
\hline P-glycoprotein & Inward facing & $\begin{array}{l}\text { 3G61 (4.35), 3G60 (4.40), 3G5U (3.80), 4F4C (3.40), 4KSC (4.00), } \\
\text { 4KSB (3.80), 4LSG (3.80), 4M1M (3.80), 4M2S (4.40), 4M2T (4.35), 4KSD (4.10) }\end{array}$ & {$[47,76,193,194]$} \\
\hline $\mathrm{CmABCB} 1$ & Inward facing & 3WME, 3WMF, 3WMG $(2.40 / 2.60)$ & [93] \\
\hline
\end{tabular}

* Numbers in brackets indicate structure resolution in $\AA$.

contain 10 transmembrane helices in each half transporter, for a total of 20 hydrophobic helices in a full transporter [36]. Both Type I and Type II importers present shorter $\alpha$-helices than the known ABC exporters and, at least the bacterial ones, require the presence of extracytosolic accessory proteins involved in substrate loading [58-60].

In a similar manner as the $A B C$ "exporters", $A B C$ "importers" have been crystallized both in "open-inwards" and "open-outwards" conformations, related to each order by rotation of the transmembrane domains as a consequence of the formation of a closed dimer between the NBDs [37]. Dimerization may be the result of ATP binding and its disruption a consequence of ATP hydrolysis. Coordination of the TMDs and the NBDs is achieved by the presence of two coupling helices extending from the TMDs of each half transporter. The coupling helices contain the so-called "EAA motif", a sequence of 15-20 amino acid residues considered to be a signature motif for ABC "importers" [61] The EAA motif interacts with several residues in the NBDs, which are specific for Type I or Type II importers [62].

At least two ABC "importers", Candida albicans Cdr3p and mammalian $A B C A 4$, appear to function as pumps catalyzing inward phospholipid transport [63-65]. Interestingly, in contrast to Cdr3p, two other members of the C. albicans Cdr family, Cdr1p and Cdr2p, have been characterized as outward phospholipid transporters [63]. Cdr3p possess 56 and 55\% sequence identity to Cdr1p and Cdr2p, respectively, and based on secondary structure predictions, would be expected to fold similarly to these and other Cdr proteins [66]. Likewise, human ABCA4 and its closest homologue, ABCA1, have a similar predicted architecture, but transport phospholipids in opposite directions [65]. The transport activity of both ABCA1 and ABCA4 was demonstrated after reconstitution of purified proteins in proteoliposomes in the absence of accessory substrate binding proteins, which provides strong evidence that these proteins are directly catalyzing phospholipid flipping in opposite directions $[64,65]$. Does this mean that Cdr3p and ABCB4 present an "importer" fold in contrast to their homologues, Cdr1p and ABCA4, respectively, which would be predicted to present "exporter" folds? Membrane span predictions and whole sequence homology show that the architecture for these importers is not compliant with the bacterial importer type but, notably, ABCA4 contains a conserved EAA amino acid motif that is a signature for bacterial $A B C$ importers $[61,67]$. It is possible that eukaryotic ABC "importers" present the same fold as "exporters", but have evolved an inverse affinity switch that allow them to transport the substrate inwards. Indeed, it has been demonstrated that some plant ABC transporters are capable of switching between the importer and exporter mode depending on substrate concentrations [68,69].

Of the five ABC "exporters" crystalized as full proteins to date, two have been shown to transport fluorescent phospholipid analogs in reconstituted systems (Tables 1 and 3) [70-72]. Due to the scarce information available about eukaryotic phospholipid "importers", in the following we will only discuss the putative transport mechanism of $A B C$ "exporters". For a more concise review on the structural folding of $A B C$ importers and its mechanistic consequences the reader is referred to $[36,73]$.

\subsubsection{Putative catalytic mechanism of $A B C$ transporters of the "exporter" fold}

The precise mechanism of coupling ATP binding and hydrolysis to transport is still not completely understood. Two general models try to explain this process: (i) the "ATP switch" model, and (ii) the "constant contact" model. Common for both is that they are "alternating access" models, according to which the pump alternates between two major conformational states. In one state, the binding site for the transported substrate faces one side of the membrane. In the other state, the site faces the other side of the membrane. A number of other proposals have been made that provide intermediate solutions for the mechanism of transport of some $A B C$ exporters. The reader is referred to [53] for an extensive discussion on the subject.

According to the first "ATP switch" model (Fig. 1B) [25,74], the NBDs fully disassociate from each other during the catalytic cycle. Each catalytic cycle starts with the TMDs in an open-inward conformation and the NBDs separated from each other and none of them with bound nucleotide. The high affinity of the binding site will favor binding of the substrate inside the cavity. Substrate binding results in a rearrangement of the TMDs and repositioning of the NBDs, which allows for ATP binding at both sites and formation of a closed-dimer intermediate. According to this model, binding of ATP and subsequent movement of the NBDs into the closed-dimer configuration provides the power stroke that forces the transmembrane segments of the TMDs to close the cavity facing the cytoplasm and assume an open-outwards conformation. Rearrangement of amino acid residues with respect to the substrate during movement of the TMDs eliminates the high affinity binding site, which has the consequence that the substrate gets released to the extracellular side of the membrane. Finally, hydrolysis of either one or both molecules of ATP disrupts the closed NBD dimer and restores the $\mathrm{ABC}$ transporter in its original configuration. The driving force for this reaction comes from an increase in negative charge at the NBD due to the formation of ADP and Pi with subsequent electrostatic repulsion [75].

Although the ATP switch model can explain the action of many ABC transporters, it is still highly debated. Recent crystallographic characterization of human ABCB10 and the heterodimeric transporter TM287/ 288 from Thermotoga maritima suggests that the two NBDs are never far apart from each other during the catalytic cycle $[40,48]$, in contrast with what was indicated by the apo-structures of MsbA and Pglycoprotein $[39,47,76]$. Moreover, it is still unclear whether it is ATP binding or ATP hydrolysis that actually drives conformational changes accompanying substrate translocation. The crystal structures of bacterial Sav1866 and MsbA in the presence of the non-hydrolysable ATP analog AMP-PNP present an open-outwards conformation [39,45]. This would suggest that ATP binding is sufficient to stabilize the open outwards conformation implying that the power stroke for conformational change happens before ATP hydrolysis. In contrast, crystallization of human ABCB10 and the heterodimeric Thermotoga transporter TM227/228 in the presence of the same ATP analog results in proteins locked in the open-inwards conformation $[40,48]$, which in analogy with the 
assumption above would indicate that ATP hydrolysis is required for the large conformational change. Whether these differences are due to mechanistic reasons or just an artifact of crystallization remains to be elucidated. Indeed, molecular dynamics simulations with human ABCB1 have led to the proposal that the very wide open-inwards conformations may represent non-physiological states that are stabilized by crystal lattice contacts [77].

According to the second "constant contact model", the two NBDs do not dissociate from each other during catalysis, and each ATP binding site hydrolyze ATP and open alternately $[78,79]$. This implies that a single catalytic cycle does not imply binding, hydrolysis and release of the same ATP molecule. Instead, in each transport cycle, one of the NBDs will hydrolyze a bound ATP, while the other NBD will unload ADP and Pi produced at this site during the previous catalytic cycle. In order to promote ADP and Pi release, the NBD containing hydrolyzed ATP rotates outwards which in turn forces the pump to acquire an inward-open conformation, as shown in the crystal structures of MsbA. The proposal that the NBDs never become distantly separated from each other is supported by the ABCB10 structures [40]. The biochemical and biophysical evidence supporting the "constant contact model" has been reviewed recently [80].

$A B C$ "exporters" seem to have a stoichiometry of one to two molecules of ATP hydrolyzed per molecule of transported substrate. However, it has been shown that some of these transporters can be partially uncoupled in reconstituted systems [81,82]. A quest to understand the uncoupling process has led to extensive evidence that the two NBDs bind and hydrolyze ATP in a cooperative manner [83-86].

\subsubsection{Putative phospholipid flipping mechanism of $A B C$ transporters of the "exporter" fold}

Although many details are known about the mechanism by which ABC exporters carry out ATP hydrolysis and transduce the rearrangement of the NBDs to the TMDs, very little is known about transport of the substrate. Of the five $A B C$ transporters of the exporter fold crystallized to date only bacterial MsbA and P-glycoprotein (ABCB1) have been shown to transport lipidic substrates. Depletion of cellular MsbA or the presence of a conditionally inactivating mutation in this protein result in loss of lipid $A$ and phospholipid transport from the cytoplasmic to the outer membrane in bacterial cells, suggesting a general flippase function for MsbA [87,88]. This evidence is confirmed by reconstitution experiments, in which MsbA has been shown to translocate a wide variety of fluorescent-labeled phospholipids, albeit with low activity [72]. In the case of P-glycoprotein, studies in cell lines and reconstituted systems have suggested the involvement of this protein in transport of a variety of phospho- and glycolipids [65,70,89-91]. However, this protein was first identified as the multidrug resistance transporter responsible for the inefficiency of long-term pharmacological treatments [92]. The flexibility in the recognition of substrates complicates greatly the task of defining a putative binding site for phospholipids within one of these crystal structures. Furthermore, most crystal structures for $A B C$ "exporters" have a relatively low resolution (Table 3 ), which do not allow for confident modeling of the position of the amino acid chains. It has not been until very recently that the barrier of 3 Å resolution was broken and a putative entry pathway for an amphipathic substrate was proposed for the mitochondrial putative di-peptide transporter $A B C B 10$ [40]. Recently, the crystal structure of an inward open half-size multidrug transporter, CmABCB1, was solved at $2.6 \AA$ resolution, which, supported by mutant and transport analyses, revealed the structural basis for drug substrate gating [93]. Interestingly, a cavity lies exactly within the hydrophilic heads of the lipids and corresponds to the MsbA stretch where Cys mutants showed decreased basal activity [94].

Two speculative models on ABC-type flippases have been proposed, the "tilting model" and the "rotating helix model", that are however not exclusive $[93,216]$. In the tilting model, a variation of the alternating access model, the lipid enters the chamber of MsbA from the inner leaflet of the bilayer. ATP binding causes conformational changes of the TMDs, leading to the release of the substrate into the outer space (see Fig. 1B). In the rotating helix model, conformational changes of $\mathrm{ABCB} 1$ lead to the rotation of the TM helices reorienting the substrate-binding site from the inner leaflet of the lipid bilayer to the outer, from where the substrate is released.

Data from the $A B C B 1$ structures revealed substantial TM helices repacking upon nucleotide binding, which is consistent with the rotating helix model [95]. However, in respect to the significant size of most lipids but especially the lipid-A core transported by MsbA, it seems likely that the flippase $A B C$ does not completely enclose the lipid substrates, which is better covered by the alternating access model. This has been widely accepted for other flippases and is further supported by structural data on MsbA employing spin labeling and EPR [94]. However, the finding that both crystal structure and EPR studies indicate large conformational changes in MsbA that correlate with the huge size of the MsbA lipid-A substrate, might simply indicate that MsbA and ABCBs employ distinct flippase mechanisms.

Mutations in phospholipid binding domains should have a striking impact on the activity and substrate specificity of flippases. Site-directed mutagenesis has been carried out extensively in MsbA and substitution of a number of residues affected catalytic turnover by this transporter [94]. However, as only basal ATP hydrolytic activity was determined, it remains to be shown that in this system ATP hydrolysis is strictly coupled to transport. Interestingly, the suggested lipid-drug duality of flippases has been explained by a coupling of phospholipid transportation and drug transport [96]. According to this assumption ABCB1 would act as a phospholipid flippase caused as a side effect of its main drug transport function, while the opposite would hold true for MsbA [97].

In all $A B C B 10$ structures, TM1-3 and TM6 of one monomer associate with TM4 and TM5 of the other monomer [40]. In the structure of the ABCB10 homodimer in the absence of bound nucleotide, TM1 and TM2 are separated from each other by a maximal distance of almost $14 \AA$, enough for an amphipathic substrate to be bound between the transmembrane loops. This distance is kept in a structure in which the NBDs are bound to the non-hydrolysable ATP analog AMP-PCP (Fig. 2A). Interestingly, the latter structure shows some electron density in the region between TM1 and TM2 that can be modeled to the aliphatic chain of a cardiolipin molecule (Fig. 2A). Although ABCB10 has never been implicated in the transport of cardiolipin, the structure demonstrates the possibility for the aliphatic chain of a lipid-like amphipathic substrate to penetrate sideways from the membrane into the TMD of the $\mathrm{ABC}$ exporter. Sequence homology alignments between different members of the $A B C B$ family of $A B C$ transporters have identified the presence of highly conserved residues in TM1-3 that have been suggested to form part of the substrate binding region (Fig. 2A) [40]. These residues are located toward the cytosolic side of the large central cavity of the ABC dimer where they have been proposed to coordinate the hydrophilic part of an amphipathic substrate, whereas its hydrophobic region could be accommodated in the gap formed by TM1 and TM2 (Fig. 2A). In a third structure, crystallized in the presence of the ATP analog AMP-PNP, the distance between TM1 and TM2 is reduced to about $9 \AA$, which suggests a conformation wherein the entry pathway is closed to prevent the substrate from moving back into the membrane before transport to the other leaflet takes place [40].

The proposed transport mechanism of $A B C B 10$ is in agreement with the rotating helix model, in which rotation of the TMDs during the catalytic cycle allows for alternating opening not only of a central cavity, but also of lateral entry and exit pathways for the transported phospholipid substrate [95]. Unfortunately, all structures obtained for ABCB10 are in an open-inwards or closed-inwards conformation with the TMDs forming a central cavity opened to the cytosolic side, and the absence of a structure in the proposed open-outwards conformation limits our understanding of the catalytic cycle and how the transported substrate is delivered back to the membrane. However, although the AMP-PNP bound form of ABCB10 presents a closed-inwards conformation, the AMP-PNP-bound forms of the bacterial "exporters" MsbA and 
Sav21866 show an open-outwards conformation, where the TMDs rearrange to create a substantial central cavity opened to the exoplasmic side of the membrane (Fig. 2A). TM1 and TM2 for one monomer, now associate with TM3-TM6 of the other monomer. TM3 and TM4 are now forming a lateral opening analogous to the one formed by TM1 and TM2 in the open-inwards conformation of ABCB10, which would allow for lateral exit to the membrane environment of a transported amphipathic substrate. Further biochemical and structural characterization is required to understand whether these gaps between transmembrane domains actually form pathways for entry and exit of the substrate.

\subsection{Phospholipid flippases of the P-type ATPase family}

P4-ATPases constitute a major group of phospholipid flippases that are not related to $A B C$ transporters. Rather, they belong to the P-type ATPase superfamily, a large family of ATP driven biological pumps, which transport a number of different substrates but share the common feature that they form a phosphorylated intermediate during their catalytic cycle [98]. P-type ATPases are divided into five subfamilies (P1 to P5) characterized by their transported substrate [98]. P1 to P3type ATPases are cation transporters, such as the heavy metal transporters involved in cytoplasm detoxification and loading of metals inside specific tissues and cellular compartments (P1-ATPases), the sarcoendoplasmic reticulum $\mathrm{Ca}^{2+}$-ATPase (SERCA; a P2-ATPase) involved in maintaining calcium homeostasis during signaling, the plasma membrane $\mathrm{Na}^{+} / \mathrm{K}^{+}$-ATPase (a P2-ATPase) that generates the membrane potential in mammalian cells, and the plasma membrane $\mathrm{H}^{+}$-ATPase energizing plant and fungal cell membranes (a P3ATPase). Recently, structured logistic regression has been used as a machine approach to identify all P-type ATPases in the UniProtKB database and classify them in P-type ATPase subfamilies [99]. Using this automated method less than one hundred prokaryotic sequences can with low statistical significance be classified as P4- and P5-ATPases [95], but individual inspection of the sequences identified so far reveals that they all show higher overall homology to P2-ATPases than to P4-ATPases. We can thus conclude that while cation transporters of the P-type family are found in all three branches of life, members of the P4- and P5ATPase subfamilies are only present in eukaryotic organisms. Whereas the transported substrate of P5-ATPases remains to be determined, P4-ATPases act as inward phospholipid transporters (Table 2).

\subsubsection{General structural features of P-type ATPases}

In contrast to $\mathrm{ABC}$ transporters, the catalytic subunit of P-type ATPases is always encoded by a single polypeptide (Fig. 1C). Several crystal structures are available that allow for visualization of various pump proteins at different steps during the catalytic cycle (Table 4). Even though the published P-type ATPase crystal structures show characteristic individual peculiarities, they all exhibit a common fold. Thus, four distinct structural regions, first defined and named by Toyoshima et al. (2000) [100], are present in all P-type ATPases (Fig. 1C): a membrane domain $(\mathrm{M})$, that forms the pore through which the substrate is transported; a variable nucleotide-binding domain $(\mathrm{N})$, that functions as a built-in protein kinase that phosphorylates the pump and where ATP docks mainly through hydrophobic stacking of the adenosine ring to a conserved phenylalanine; a phosphorylation domain $(\mathrm{P})$ that contains the conserved amino acid sequence DKTG including the aspartate residue that gets phosphorylated during catalysis (hence P-type) and two other conserved motifs TGDN and GDGXND involved in the coordination of a $\mathrm{Mg}^{2+}$ ion required for ATP binding at the phosphorylation site; and a built-in protein phosphatase, named the actuator domain (A), comprising the amino acid sequence TGE, in which the glutamic acid generates a nucleophilic attack on the phosphorylated aspartate and causes dephosphorylation of the P-type pump. These four regions are connected to each other with amino acid linkers and suffer dramatic movements during the catalytic cycle, which involves two main protein conformations, Enzyme 1 (E1) and Enzyme 2 (E2), in which the substrate binding site presents different substrate affinities and is open to opposite sides of the membrane (Fig. 1D).

The conformational transitions between E1 and E2 are large and require very flexible pump molecules. For some P-type ATPases, the $\mathrm{N}$ - and/or C-terminal domains are autoinhibitory, preventing the conformational changes required for substrate transport by establishing intramolecular interactions with other parts of the P-type ATPase $[101,102]$. These autoinhibitory domains contain regulatory sequences that interact with accessory proteins, such as calmodulin or 14-3-3 proteins in order to trigger activation of the pump [103].

Like other P-type ATPases, P4-ATPases are predicted to have 10 transmembrane spanning segments and both $\mathrm{N}$ - and $\mathrm{C}$-termini facing the cytosol [104]. The A-domain is formed by amino acid residues located at the N-terminal domain and the intracellular loop between TM2 and TM3, while the P- and N-domains are located to the intracellular loop between TM4 and TM5 [10399].

\subsubsection{General catalytic cycle of P-type ATPases}

The SERCA1 $\mathrm{Ca}^{2+}$ pump from rabbit skeletal muscle is by far the best structurally characterized P-type ATPase. Not only E1 and E2 forms of the protein have been crystallized, but also a large number of intermediate conformations representing almost all possible protein movements and interactions with the transported substrate, ATP or its hydrolysis products (Table 4 ). Taken together, this wealth of structural information makes it possible to outline a detailed mechanism for the action of this pump [105].

The catalytic cycle of cation transporting P-type ATPases starts when the protein in the E1 conformation binds the substrate at the M-domain in a high affinity site opened to the cytosolic side of the membrane [106, 107]. This event initiates a structural rearrangement that is transmitted across the pump molecule and makes possible the phosphorylation of the P-domain by an ATP molecule already bound at the N-domain, generating the E1P conformation. Transition into the E2P state occurs when the A-domain rotates almost $120^{\circ}$ and forces a reorganization of the M-domain, which brings the active site of the built-in protein phosphatase of the A-domain in close proximity to the phosphorylated aspartyl group. This reorganization results in an initial occluded state in which the ion is blocked inside the protein and, subsequently, the opening of a low affinity binding site to the exoplasmic side of the membrane, which causes release of the bound cytosolic substrate and, in some cases, binding of a counter-ion. Nucleophilic attack of the Adomain onto the phosphorylated aspartate leaves the protein in the $\mathrm{E} 2$ conformation that is unstable and transitions to the $\mathrm{E} 1$ conformation forcing the A-domain to rotate back to its original position. Movement of the A-domain results in reorganization of the M-domain, with the consequent release of the exoplasmic substrate (the counter-ion) to the cytosolic side of the membrane, see [108] for further detail.

Although no crystal structure is available for a P4-ATPase, these proteins are probably not very different in folding from other members of the P-type ATPase family, as suggested by the fact that (i) all P-type ATPases including P4-ATPases have conserved core sequence segments that are essential for catalysis [98,109], and (ii) all crystallized P-type ATPases show a very similar tertiary structure despite their varying TMD numbers and diverse substrates ranging from protons to heavy metals $[108,110]$. Thus, assuming an analogous transport mechanism for P4-ATPases as for the SERCA pump, the phospholipid would occupy the place of the counter-ion and therefore be bound in the E2P conformation at the exoplamic side of the membrane and released in the transition step to $\mathrm{E} 2$ on the cytosolic side. Whether an additional substrate is transported in the opposite direction is yet unknown.

\subsubsection{Putative phospholipid flipping mechanism of P4-ATPases}

In contrast to the different cations transported by members of the P1 to P3 subfamilies of P-type ATPases, phospholipids are voluminous substrates that contain both a substantial hydrophilic head group and two 


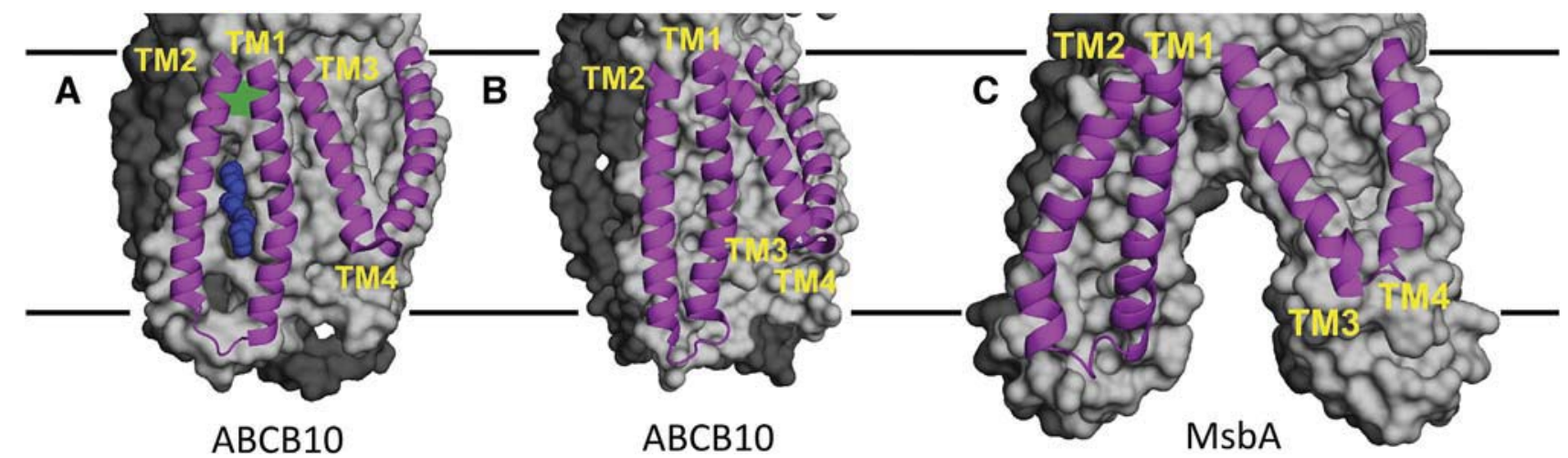

Fig. 2. Putative lipid translocating pathways for an ABC exporter. (A) In the Rod A crystal structure of ABAB10 (PDB code 4AYT), a lateral opening between TM1 and TM2 shows electron density that can be assigned to a cardiolipin molecule (dark blue). A putative lipid would be loaded by inserting its fatty acid tail in an analogous manner, while the head group interacts with hydrophilic residues (green). (B) A closing of the gap between TM1 and TM2 results in occlusion of the lipid as suggested by the plate form crystal structure of ABCB10 (PDB code 4AYW). (C) Based on the crystal structure of MsbA in the presence of AMP-PNP (PDB code 3B60), rotation of the alpha helices in the TMD generates a lateral opening to the membrane between TM3 and TM4 that can be used to unload the lipid.

long fatty acid chains of a hydrophobic nature (Fig. 3C). It is difficult to envisage how such a substrate can be introduced inside a protein pore opened to one side of the membrane, be entirely occluded during the structural transition of the protein through the catalytic cycle, and then exposed to the other side of the membrane for its release. So how do P4-ATPases manage their substrate?

A characteristic feature of P4-ATPases is the presence of a $\beta$-subunit required for the catalytic cycle. This protein belongs to the family of yeast Cdc50 proteins and contains two transmembrane domains and a large luminal loop that is heavily glycosylated [111] (Fig.1C). Topologically, this protein resembles a fusion of the $\beta$ - and $\gamma$-subunits of mammalian $\mathrm{Na}^{+} / \mathrm{K}^{+}$-ATPases [112]. As in the case of the $\mathrm{Na}^{+} / \mathrm{K}^{+}$-ATPase $\beta$-subunit, the Cdc50 protein is required for proper folding and exit of the P-type ATPase from the ER [111,103-116]. For the $\mathrm{Na}^{+} / \mathrm{K}^{+}-$ ATPase, the $\gamma$-subunit is involved in the modification of the kinetic parameters of cation transport depending on tissue and environmental conditions [117-119]. For the yeast P4-ATPases, the Cdc50 protein is strictly required for the generation of a phosphorylated intermediate and consequently for phospholipid transport, although it is not clear how this protein contributes to P4-ATPase activity [120,121]. It has been suggested that the Cdc50 protein is interacting with the transported phospholipid and responsible together with the P-type ATPase of generating a pathway for movement of the phospholipid through the membrane [121]. However, phospholipid specificity determinants for the P4-ATPase/ $\beta$-subunit complex reside solely in the P-type protein $[114,122]$, which makes it improbable for the $\beta$-subunit to directly participate in substrate binding during transport. Most likely, the $\beta$-subunit is required for stabilizing the P4-ATPase in some of the intermediate conformations during catalysis [123].

Recent mutational studies suggest two pathways by which P4ATPases could transport their substrate (Fig. 3): (i) the canonical pathway with the phospholipid transported through the interior of P4-ATPases in analogy with the cation transport mechanism of well characterized P2-ATPases and (ii) a non-canonical pathway at the protein-membrane interface. An increasing amount of biochemical evidence seems to suggest that these two extreme models might be partial descriptions of the same mechanism.

In the thoroughly studied $\mathrm{Ca}^{2+}$ - and $\mathrm{Na}^{+} / \mathrm{K}^{+}$transporting P2-ATPases, the cation binding sites are present in small central cavities primarily formed by charged or polar residues in the center parts of transmembrane segments TM4, TM5, and TM6 [105]. Conserved acidic group(s) positioned in the middle of TM4 and TM6 secure charge neutralization of the transported cation, while two conserved asparagine residues in TM5 and TM6 provide oxygen groups for ion coordination. Further coordination is provided by backbone carbonyl oxygen atoms in TM4, that contains one or more highly conserved proline residues that function as helix-breakers [103]. Likewise, all P4-ATPases contain a conserved proline in the middle of TM4 (Pro-507 in Drs2p) suggesting the presence in these pumps of a central cavity with carbonyl oxygens contributing to the coordination of a hydrophilic group.

In contrast, P4-ATPases do not have preserved acidic residues in their predicted transmembrane segments [109]. However, a basic lysine residue (corresponding to Lys-1018 in the yeast P4-ATPase Drs2p; Lys873 in the mammalian photoreceptor P4-ATPase ATP8A2) is conserved in all P4-ATPases and situated in the middle of TM5 at the same position as cation-coordinating residues in P2-ATPases [124]. Mutation of this residue has a dramatic negative impact on the apparent affinity of ATP8A2 for its substrate PS as judged by the ability of the substrate to induce dephosphorylation, or stimulate ATPase activity [124]. The binding energy of the substrate has to be transduced to the cytosolic domains in these assays, and either the binding or transduction could be perturbed by the mutation [124]. Given that several P4-ATPases tolerate drastic modifications of the acyl chains of the phospholipid substrate, this model would imply considerable flexibility of P4-ATPases to accommodate their substrate and especially the phospholipid tail when it traverses the membrane.

In contrast to this, substitution in the yeast P4-ATPase Dnf1p of the conserved lysine in TM5 (Lys-1194) results in a reduction of protein activity, but does not affect substrate specificity, suggesting that this residue is not directly binding the substrate [125]. Further mutational studies of Dnf1p identified amino acid residues involved in substrate selection at the lumenal side of TM1 and the cytosolic sides of TM1, TM2, TM3 and TM4, but not within the membrane region [122,125]. Two main residues determine the specificity of the Dnf1p protein: a tyrosine close to the cytosolic side of TM4 (Tyr-618 in Dnf1p) and a phenylalanine in the loop that connects TM3 and TM4 (Phe-587 in Dnf1p). Mutation of Tyr-618 to phenylalanine (the corresponding residue in the PS-transporting P4-ATPase Drs2p) confers Dnf1 the ability to transport PS, without affecting PC and PE translocation, while mutation of Phe587 reduces PC recognition without affecting PE. This feature has led to the proposal of a two-gate system that would recognize the phospholipid on one side of the membrane, allow it to slide to the other side with the hydrophobic tail always protruding through a cleft toward the phospholipid bilayer and then release it on the other side of the membrane [125].

Indeed, a combination of biochemical analysis of mutants and protein modeling has been recently used to suggest a mechanistic model in which a conserved isoleucine in TM4 (corresponding to Ile-364 in the mammalian photoreceptor P4-ATPase ATP8A2) and its adjacent residues form a hydrophobic gate that allows the formation of alternating water-filled cavities on both sides of the groove formed by TM1, TM2, TM4, and TM6, similarly to the cation-gating role ascribed to the 
Table 4

Available crystal structures for P-type ATPases.

\begin{tabular}{|c|c|c|c|c|}
\hline Protein & Subclass & Conformation* & PDB entry** & Reference \\
\hline \multirow[t]{2}{*}{$\mathrm{Na}^{+}, \mathrm{K}^{+}$-ATPase } & \multirow[t]{2}{*}{ P2 } & E2P & $\begin{array}{l}\text { 3N2F (4.10), } 3 \text { N23 (4.60), 3B8E (3.50), 3KDP (3.50), } \\
\text { 3A3Y (2.80), 2ZXE (2.40) }\end{array}$ & [195-197] \\
\hline & & E1P & 4HOJ (4.30), 3WGU(2.80), 3WGV (2.80) & {$[198-200]$} \\
\hline $\mathrm{H}^{+}$-ATPase (AHA2) & P3 & E1 & $3 \mathrm{~B} 8 \mathrm{C}(3.60)$ & {$[110]$} \\
\hline $\mathrm{Cu}^{+}$-ATPase (CopA) & P1 & E2P & 3RFU (3.20), 4BBJ (2.80) & {$[201,202]$} \\
\hline \multirow[t]{4}{*}{$\mathrm{Ca}^{2+}$-ATPase (SERCA) } & \multirow[t]{4}{*}{ P2 } & E1 & 4H1W (3.10), 3W5A (3.01), 3W5B (3.20), 3TLM (2.95), 2C9M (3.00) & [203-206] \\
\hline & & E1P & $\begin{array}{l}\text { 3BA6 (2.80), 3N8G (2.59), 2DQS (2.50), 1VFP (2.90), 1T5S (2.60), 1T5T (2.90), } \\
\text { 1SU4 (2.40) }\end{array}$ & {$[100,128,207-211]$} \\
\hline & & E2P & $\begin{array}{l}\text { 4J2T (3.20), 3N5K (2.20), 3AR2 (2.50), 3AR3 (2.30), 3AR4 (2.15), 3AR5 (2.20), } \\
\text { 3AR6 (2.20), 3AR7 (2.15), 3AR8 (2.60), 3AR9 (2.60), 3B9B (2.65), 3FGO }(2.50) \text {, } \\
\text { 3FPB (2.55), 3FPS (3.20), 3N23(4.60), 3W5D (2.45), 2ZBD (2.40), 2ZBE }(3.80) \text {, } \\
\text { 2ZBF (2.40), 2ZBG (2.55), 209J (2.65), 1XP5 (3.00), 1WPG (2.30) }\end{array}$ & $\begin{array}{l}{[129,133,196,204,207} \\
209-214]\end{array}$ \\
\hline & & E2 & 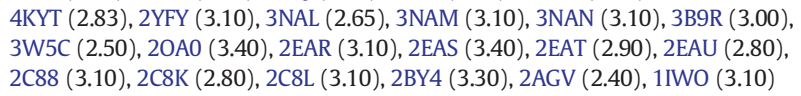 & {$[128,129,204,206,214-220]$} \\
\hline
\end{tabular}

\footnotetext{
* E1 and E2 denote totally dephosphorylated pumps, while E1P and E2P indicate the phosphorylated states and the transitions states to/from dephosphorylated conformations.

** Numbers in brackets indicate structure resolution in $\AA$.
}

conserved glutamate residue present at the same position in other P-type ATPases [126]. These water-filled cavities would protect the hydrophilic phospholipid head group from the hydrophobic environment during its passage through the membrane, while the phospholipid tails protrude freely into the hydrophobic environment.

Further studies on the cooperation of Tyr-618 with other residues of TM1-TM3 has demonstrated that a number of amino acid residues located on the cytosolic side close to the M-domain contribute to a proposed exit gate for the phospholipid [127]. These residues also allow Dnf1p (and its homologue Dnf2p) to discriminate between mono- and di-acylphospholipids, which lead to the proposal that these yeast P4ATPases function primarily as lyso-lipid transporters [127]. Based on homology modeling using the $\mathrm{Na}^{+} / \mathrm{K}^{+}$-ATPase as a template, these residues were mapped to a region of the SERCA $\mathrm{Ca}^{2+}$-ATPase that forms a binding pocket for a PE molecule in the E2 conformation [127, 128]. This PE molecule has been proposed to assist $\mathrm{Ca}^{2+}$ entry into a deep, funnel-shaped and negatively charged path that leads to the $\mathrm{Ca}^{2+}$ coordination sites located halfway through the membrane bilayer $[128,129]$. The binding pocket is not accessible in the E1 conformation, implying that the PE molecule has to dynamically enter and exit the pocket as the SERCA $\mathrm{Ca}^{2+}$-ATPase proceeds through the catalytic cycle. It is possible that this original phospholipid head group binding pocket and cation-loading funnel have evolved in P4-ATPases to generate a cleft for phospholipid translocation. Indeed, the homology model for Dnf1p in the E2 conformation shows a triangular shaped groove flanked by TM1 and TM3 on the sides and TM4 on the back [122]. Although the Dnf1p structural model has proven useful to identify residues for biochemical studies, conclusions based on it need to be carefully assessed, as the protein shows only $17 \%$ identity to the $\mathrm{Na}^{+} /$ $\mathrm{K}^{+}$-ATPase used as a template. Further, the model cannot explain some of the most recent mutagenesis results. Two Dnf1p residues located inside the membrane plane in TM2 (Thr-254 and Asp-258) are involved in discrimination of lysophospholipids [127]. However, in the model, TM2 is located behind TM1 and has no access to the groove formed by TM1, TM3 and TM4, where the phospholipid is suggested to bind.

Interestingly, SERCA has been crystalized in the E2 conformation with bound thapsigargin, a potent inhibitor of the SERCA pump with phospholipid like features (Fig. 4, in blue) [128]. This crystal structure also shows electron density that can be assigned to a PE molecule (Fig. 4, in green). While PE is localized in a groove formed by TM2 and TM4 with TM1 covering the backside, thapsigargin binds in a similar groove on the opposite side of the M-domain, with TM3 and TM6 forming the sides and TM5 at the back. Notably, the acyl chains of thapsigargin sit deeply between the transmembrane sections of the
SERCA $\mathrm{Ca}^{2+}$-pump, which would be consistent with high structural flexibility [128]. Whether the position of the transmembrane segments in P4-ATPases would allow for binding of a phospholipid (and its subsequent transport) in a position analogous to that of PE or tharpsigargin in SERCA still needs to be demonstrated and would require the solution of a crystal structure for one of these proteins. However, it seems possible that P4-ATPases have evolved to generate a pathway through the membrane that would connect both binding sites, allowing for phospholipid movement across them.

\subsection{Comparison of ABC transporters of the "exporter" fold and P4-ATPases}

From a distant point of view, ABC transporters of the "exporter" fold and P4-ATPases might look as if they were similar machines: both exist in at least two conformers with different substrate affinities at each side of the membrane and ATP provides the driving force for a conformational interchange. Generally speaking the mechanism by which both proteins hydrolyze ATP is analogous, as can be inferred by the fact that both types of proteins are sensitive to vanadate [130,131], which mimics the transition state of ATP during hydrolysis [132].

A closer look reveals major differences between the transporters. Most notably, the fate of the $\gamma$-phosphate released from the hydrolysed ATP molecule, is very different in the two pump classes: P4-ATPases form a phosphorylated intermediate in which Pi is covalently bound to an aspartate residue located in a conserved amino acid motif within the nucleotide binding site. Release of this Pi requires active nucleophilic attack of a glutamic acid in the actuator domain [133]. In contrast, covalent protein phosphorylation has not been described for ABC transporters [74]. Further, P4-ATPases have a single location for ATP binding, while ABC transporters feature a closed NBD dimer that is stabilized by ATP as described in detail above. Up to now there are no structural reports of NBDs of P-type ATPases functioning as dimers. Moreover, in $\mathrm{ABC}$ exporters, $\mathrm{ADP}$ and $\mathrm{Pi}$ are suggested to be released by electrostatic repulsions after hydrolysis [74] and therefore ATP hydrolysis is most probably not used for the translocation step but for resetting the $A B C$ transporter to its original conformation.

Despite these differences in energization, based on protein mass and structural models, the size of ABC exporters and P4-ATPases would be expected to be similar in the membrane (Fig. 1E) and the models proposed for explaining phospholipid transport by these two types of transporters present some remarkable similarities. Are $A B C$ transporters and P4-ATPases using the same mechanism to transport phospholipids in opposite directions? 

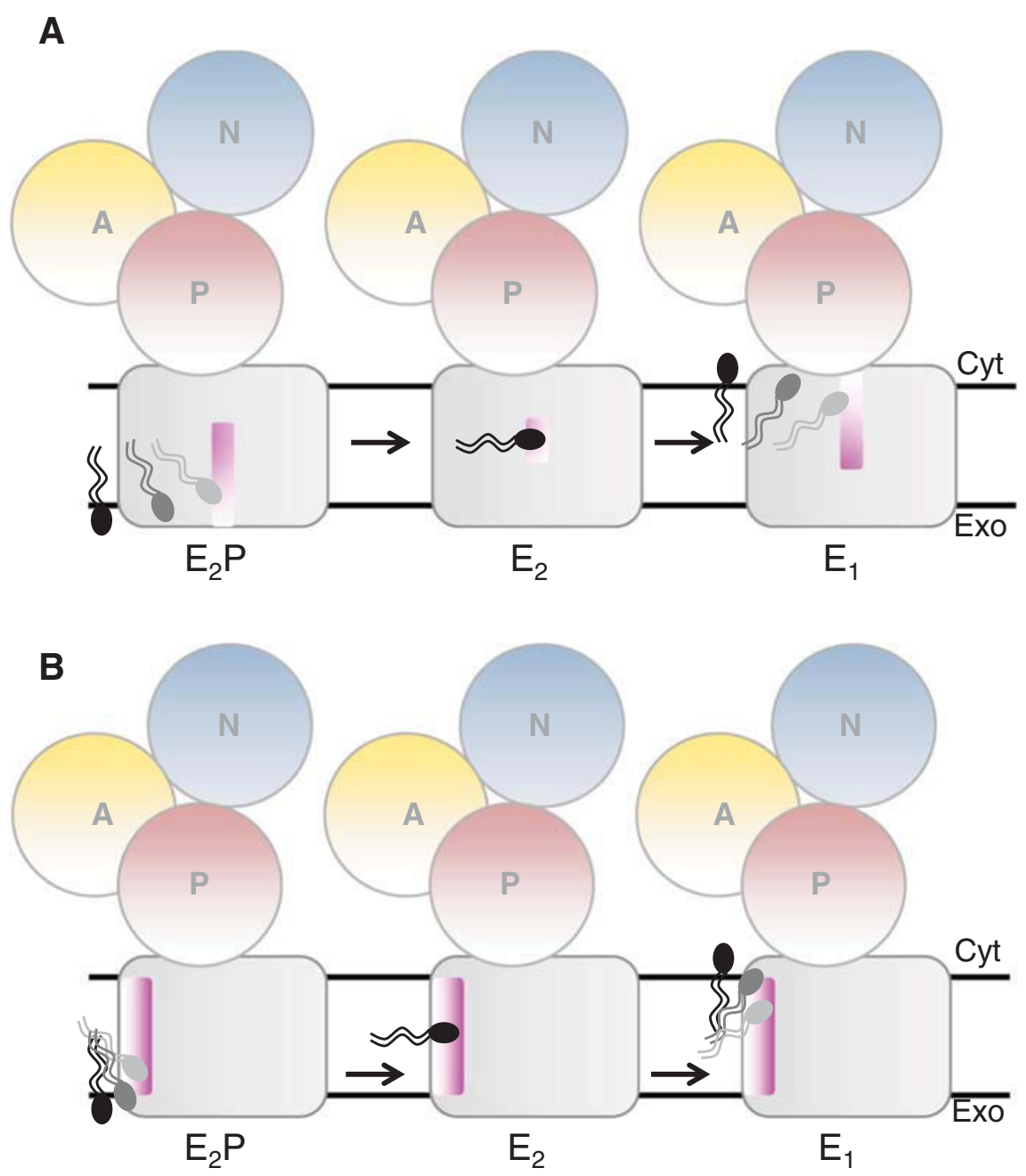

C

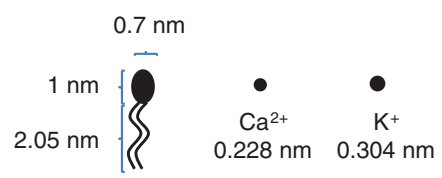

Fig. 3. The canonical versus the non-canonical model of lipid translocation by P4-ATPases. (A) The canonical pathway, in which the lipid enters the protein in a way analogous to the entry of cations in other P-type ATPases. After occlusion in the binding site, the rearrangement of the TM-domain results in opening of an exit pathway on the opposite side of the membrane. (B) The non-canonical pathway, in which the lipid head group is recognized by interaction with residues located in the boundary of the membrane, slides through the protein surface and is released on the other side of the membrane after rearrangement of the TM-domain. (C) Relative sizes of various substrates transported by P-type ATPases.

In both the "alternating access model" for ABC transporters and in the classical transport model for P4-ATPases, the phospholipid is proposed to gain access from one side of the membrane to a central cavity inside the transporter that is then closed and opened to the opposite side (Fig. 1B and 1D). The cavities supposedly used to allocate the substrate during transport would, nevertheless, be very different in size. In ABC transporters, the wide central cavity leaves plenty of space to accommodate a complete phospholipid molecule. Such an opening is unlikely to be present in P4-ATPases, yet lipids and lipid-like molecules like thapsigargin have been found deeply embedded in the crystal structure, suggesting a possible access pathway of the substrate to a central binding pocket, in analogy to other cation-transporting P-type ATPases. However, a substantial amount of evidence is still required to understand whether the big central cavity in $A B C$ transporters is used for phospholipid translocation, and whether P4-ATPases and/or ABC transporters achieve an occluded state during phospholipid transport in which the phospholipid is buried within the pump molecule.
Likewise, the substrate binding mechanism proposed for the $A B C$ transporter $A B C B 10$ is similar to the non-canonical model for P4-ATPase transport. In both, it is suggested that a lateral groove in the transmembrane region will provide access of an amphipathic substrate directly from the membrane. For P4-ATPases, this groove would open for substrate access from the exoplasmic leaflet of the membrane in the E2P phospholipid-loading conformation and to the cytosolic leaflet in the E1 conformation after phospholipid release [122]. For $\mathrm{ABC}$ exporters, the groove would open to the cytosolic leaflet in the open-inwards, substrate-loading conformation and to the exoplasmic leaflet in the open-outwards conformation [40].

Finally, although P4-ATPases only contain 10 transmembrane domains, phospholipid translocation has only been shown for members of the family that interact with a $\beta$-subunit containing two transmembrane helices. Potentially, a total of 12 transmembrane domains might be required for flippase activity. Notably, $A B C$ transporters related to phospholipid transport also contain the same number of transmembrane 


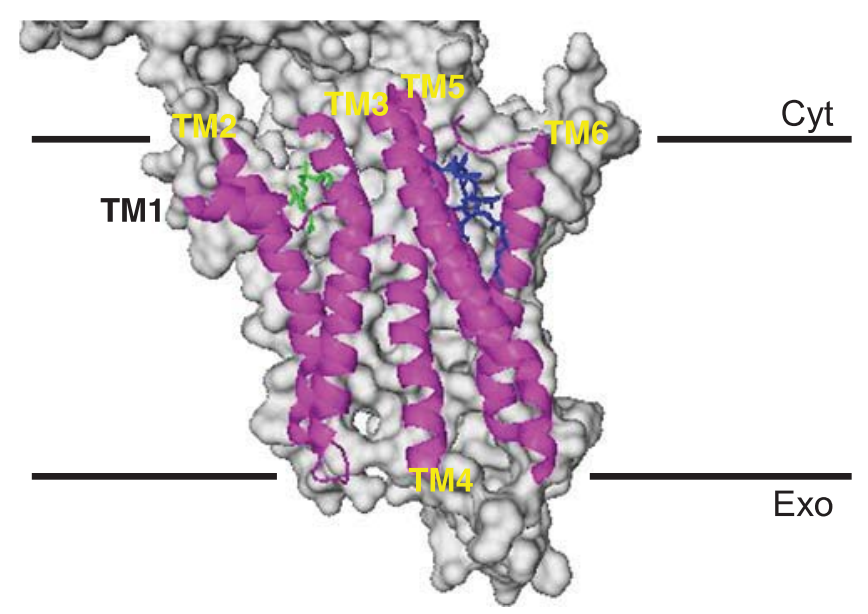

Fig. 4. Position of thapsigargin and phosphatidylethanolamine in the E2 conformation of $\mathrm{Ca}^{2+}$ pump sarcoendoplasmic reticulum calcium ATPase (SERCA1a). Thapsigargin and phosphatidylethanolamine (PE) bind in opposite sides of the SERCA E2 structure (PDB code 2AGV). PE (green) binds in a pocket formed by TM1, TM2 and TM4, while thapsigargin (blue), a molecule presenting lipid-like features, binds in a similar pocket on the other side of the protein between TM 3, TM5 and TM6.

segments. Further structural and biochemical characterization will be required to determine whether the twelve-TMD arrangement is the minimal optimized structure that allows for phospholipid translocation.

\section{Major conclusions and general significance}

Although an increasing amount of evidence is shedding light on the way phospholipid transporters discriminate their substrates, we are still very far from discerning the mechanism by which these substrates are translocated across cellular membranes. Unraveling this mechanism will require studies on the energy-dependent transporters reconstituted into proteoliposomes, a challenging task given the difficulties of purifying membrane proteins, the hydrophobic nature of the phospholipid substrates and the potential requirement for subunits and/or accessory proteins.

Promising steps have been taken to improve expression and large scale purification of P4-ATPases in complex with their subunit, a prerequisite for systematic crystallization trails [134]. Interestingly, a recent publication has reported on the ability of a P4-ATPase to counteract the toxic effects of overexpression of an $A B C$ transporter in human cells [135], suggesting that novel strategies might be required to further improve heterologous expression production for these transporters. On the other hand, technology developments in membrane protein crystallization and synchrotron microcrystallography combined with advancements in protein stabilization using lipidic cubic phases may help to obtain structural information with smaller amounts of starting material [136]. Recent progress in the functional reconstitution of ABC transporters and P4-ATPases into vesicles and nanodiscs might allow in the near future for systematic studies aiming at identifying the phospholipid binding sites and at testing the existence of an occlusion state for the substrate during transport.

Finally, most evidence on phospholipid transport is based on labeled lipid analogs. In the future, new approaches will be needed to measure transbilayer phospholipid movement using natural phospholipids, such as shape-change visualization experiments in giant unilamellar vesicles.

\section{Acknowledgments}

The authors thank the UNIK research initiative of the Danish Ministry of Science, Technology and Innovation through the "Center for Synthetic Biology" at the University of Copenhagen, the Danish National Research Foundation through the PUMPKIN Center of Excellence (DNRF85), the
Danish Council for Independent Research | Natural Sciences (FNU, grant number 10-083406), the Swiss National Funds and Novartis Foundation, the German Research Council (DFG: PO 748/10), Lundbeck Foundation (grant number R34-A3562) and the Villum Fonden (grant number 022868) for supporting their research.

\section{References}

[1] G. van Meer, D.R. Voelker, G.W. Feigenson, Membrane lipids: where they are and how they behave, Nat. Rev. Mol. Cell Biol. 9 (2008) 112-124

[2] H. Sprong, P. van der Sluijs, G. van Meer, How proteins move lipids and lipids move proteins, Nat. Rev. Mol. Cell Biol. 2 (2001) 504-513.

[3] G. van Meer, Dynamic transbilayer lipid asymmetry, Cold Spring Harb. Perspect. Biol. 3 (2011)

[4] H. Ewers, A. Helenius, Lipid-mediated endocytosis, Cold Spring Harb. Perspect. Biol. 3 (2011) a004721.

[5] T.T. Sebastian, R.D. Baldridge, P. Xu, T.R. Graham, Phospholipid flippases: building asymmetric membranes and transport vesicles, Biochim. Biophys. Acta 1821 (2012) 1068-1077.

[6] E.M. Bevers, P. Comfurius, R.F.A. Zwaal, Changes in membrane phospholipid distribution during platelet activation, Biochim. Biophys. Acta 736 (1983) 57-66.

[7] S. Krahling, M.K. Callahan, P. Williamson, R.A. Schlegel, Exposure of phosphatidylserine is a general feature in the phagocytosis of apoptotic lymphocytes by macrophages, Cell Death Differ. 6 (1999) 183-189.

[8] K. Balasubramanian, A.J. Schroit, Aminophospholipid asymmetry: a matter of life and death, Annu. Rev. Physiol. 65 (2003) 701-734.

[9] J.A. Coleman, F. Quazi, R.S. Molday, Mammalian P4-ATPases and ABC transporters and their role in phospholipid transport, Biochim. Biophys. Acta 1831 (2013) 555-574.

[10] S. Rust, M. Rosier, H. Funke, J. Real, Z. Amoura, J.C. Piette, J.F. Deleuze, H.B. Brewer, N. Duverger, P. Denefle, G. Assmann, Tangier disease is caused by mutations in the gene encoding ATP-binding cassette transporter 1, Nat. Genet. 22 (1999) 352-355.

[11] S.M. Azarian, G.H. Travis, The photoreceptor rim protein is an ABC transporter encoded by the gene for recessive Stargardt's disease (ABCR), FEBS Lett. 409 (1997) $247-252$.

[12] S.S. Strautnieks, L.N. Bull, A.S. Knisely, S.A. Kocoshis, N. Dahl, H. Arnell, E. Sokal, K. Dahan, S. Childs, V. Ling, M.S. Tanner, A.F. Kagalwalla, A. Nemeth, J. Pawlowska, A. Baker, G. Mieli-Vergani, N.B. Freimer, R.M. Gardiner, R.J. Thompson, A gene encoding a liver-specific $A B C$ transporter is mutated in progressive familial intrahepatic cholestasis, Nat. Genet. 20 (1998) 233-238.

[13] J.E. Walker, M. Saraste, M.J. Runswick, N.J. Gay, Distantly related sequences in the alpha- and beta-subunits of ATP synthase, myosin, kinases and other ATPrequiring enzymes and a common nucleotide binding fold, EMBO J. 1 (1982) 945-951.

[14] I.R. Vetter, A. Wittinghofer, Nucleoside triphosphate-binding proteins: different scaffolds to achieve phosphoryl transfer, Q. Rev. Biophys. 32 (1999) 1-56.

[15] P.C. Smith, N. Karpowich, L. Millen, J.E. Moody, J. Rosen, P.J. Thomas, J.F. Hunt, ATP binding to the motor domain from an $\mathrm{ABC}$ transporter drives formation of a nucleotide sandwich dimer, Mol. Cell 10 (2002) 139-149.

[16] J. Zaitseva, S. Jenewein, T. Jumpertz, I.B. Holland, L. Schmitt, H662 is the linchpin of ATP hydrolysis in the nucleotide-binding domain of the ABC transporter HlyB, EMBO J. 24 (2005) 1901-1910.

[17] K.P. Hopfner, A. Karcher, D.S. Shin, L. Craig, L.M. Arthur, J.P. Carney, J.A. Tainer, Structural biology of Rad50 ATPase: ATP-driven conformational control in DNA double-strand break repair and the ABC-ATPase superfamily, Cell 101 (2000) 789-800.

[18] P.M. Jones, A.M. George, Mechanism of ABC transporters: a molecular dynamics simulation of a well characterized nucleotide-binding subunit, Proc. Natl. Acad. Sci. U. S. A. 99 (2002) 12639-12644.

[19] P.M. Jones, A.M. George, Role of the D-loops in allosteric control of ATP hydrolysis in an ABC transporter, J. Phys. Chem. A 116 (2012) 3004-3013.

[20] Y.R. Yuan, S. Blecker, O. Martsinkevich, L. Millen, P.J. Thomas, J.F. Hunt, The crystal structure of the MJ0796 ATP-binding cassette. Implications for the structural consequences of ATP hydrolysis in the active site of an ABC transporter, J. Biol. Chem. 276 (2001) 32313-32321.

[21] L. Schmitt, H. Benabdelhak, M.A. Blight, I.B. Holland, M.T. Stubbs, Crystal structure of the nucleotide-binding domain of the $\mathrm{ABC}$-transporter haemolysin B: identification of a variable region within ABC helical domains, J. Mol. Biol. 330 (2003) 333-342.

[22] L.W. Hung, I.X. Wang, K. Nikaido, P.Q. Liu, G.F. Ames, S.H. Kim, Crystal structure of the ATP-binding subunit of an ABC transporter, Nature 396 (1998) 703-707.

[23] Q. Zhao, X.B. Chang, Mutation of the aromatic amino acid interacting with adenine moiety of ATP to a polar residue alters the properties of multidrug resistance protein 1, J. Biol. Chem. 279 (2004) 48505-48512.

[24] I. Carrier, I.L. Urbatsch, A.E. Senior, P. Gros, Mutational analysis of conserved aromatic residues in the A-loop of the ABC transporter ABCB1A (mouse Mdr3), FEBS Lett. 581 (2007) 301-308.

[25] J. Zaitseva, C. Oswald, T. Jumpertz, S. Jenewein, A. Wiedenmann, I.B. Holland, L. Schmitt, A structural analysis of asymmetry required for catalytic activity of an ABC-ATPase domain dimer, EMBO J. 25 (2006) 3432-3443.

[26] E. Procko, I. Ferrin-O'Connell, S.L. Ng, R. Gaudet, Distinct structural and functional properties of the ATPase sites in an asymmetric ABC transporter, Mol. Cell 24 (2006) 51-62. 
[27] R. Yang, X.B. Chang, Hydrogen-bond formation of the residue in H-loop of the nucleotide binding domain 2 with the ATP in this site and/or other residues of multidrug resistance protein MRP1 plays a crucial role during ATP-dependent solute transport, Biochim. Biophys. Acta 1768 (2007) 324-335.

[28] G.F. Ames, C.S. Mimura, S.R. Holbrook, V. Shyamala, Traffic ATPases: a superfamily of transport proteins operating from Escherichia coli to humans, Adv. Enzymol. Relat. Areas Mol. Biol. 65 (1992) 1-47.

[29] G. Schmees, A. Stein, S. Hunke, H. Landmesser, E. Schneider, Functional consequences of mutations in the conserved 'signature sequence' of the ATP-bindingcassette protein MalK, Eur. J. Biochem. 266 (1999) 420-430.

[30] Z. Szentpetery, A. Kern, K. Liliom, B. Sarkadi, A. Varadi, E. Bakos, The role of the conserved glycines of ATP-binding cassette signature motifs of MRP1 in the communication between the substrate-binding site and the catalytic centers, J. Biol. Chem. 279 (2004) 41670-41678.

[31] T.W. Loo, M.C. Bartlett, D.M. Clarke, The "LSGGQ" motif in each nucleotide-binding domain of human P-glycoprotein is adjacent to the opposing walker A sequence, J. Biol. Chem. 277 (2002) 41303-41306.

[32] X.Q. Ren, T. Furukawa, M. Haraguchi, T. Sumizawa, S. Aoki, M. Kobayashi, S. Akiyama, Function of the $\mathrm{ABC}$ signature sequences in the human multidrug resistance protein 1, Mol. Pharmacol. 65 (2004) 1536-1542.

[33] M.L. Oldham, J. Chen, Snapshots of the maltose transporter during ATP hydrolysis, Proc. Natl. Acad. Sci. U. S. A. 108 (2011) 15152-15156.

[34] P.M. Jones, A.M. George, Subunit interactions in ABC transporters: towards a functional architecture, FEMS Microbiol. Lett. 179 (1999) 187-202.

[35] C.L. Perria, V. Rajamanickam, P.E. Lapinski, M. Raghavan, Catalytic site modifications of TAP1 and TAP2 and their functional consequences, J. Biol. Chem. 281 (2006) 39839-39851.

[36] K.P. Locher, Review. Structure and mechanism of ATP-binding cassette transporters, Philos. Trans. R. Soc. Lond. B Biol. Sci. 364 (2009) 239-245.

[37] D.C. Rees, E. Johnson, O. Lewinson, ABC transporters: the power to change, Nat. Rev. Mol. Cell Biol. 10 (2009) 218-227.

[38] J. Dong, G. Yang, H.S. McHaourab, Structural basis of energy transduction in the transport cycle of MsbA, Science 308 (2005) 1023-1028.

[39] A. Ward, C.L. Reyes, J. Yu, C.B. Roth, G. Chang, Flexibility in the ABC transporter MsbA: alternating access with a twist, Proc. Natl. Acad. Sci. U. S. A. 104 (2007) 19005-19010.

[40] C.A. Shintre, A.C. Pike, Q. Li, J.I. Kim, A.J. Barr, S. Goubin, L. Shrestha, J. Yang, G. Berridge, J. Ross, P.J. Stansfeld, M.S. Sansom, A.M. Edwards, C. Bountra, B.D. Marsden, F. von Delft, A.N. Bullock, O. Gileadi, N.A. Burgess-Brown, E.P. Carpenter, Structures of ABCB10, a human ATP-binding cassette transporter in apo- and nucleotide-bound states, Proc. Natl. Acad. Sci. U. S. A. 110 (2013) 9710-9715.

[41] R.J. Dawson, K.P. Locher, Structure of a bacterial multidrug ABC transporter, Nature 443 (2006) 180-185.

[42] P. Zou, M. Bortolus, H.S. McHaourab, Conformational cycle of the $\mathrm{ABC}$ transporter MsbA in liposomes: detailed analysis using double electron-electron resonance spectroscopy, J. Mol. Biol. 393 (2009) 586-597.

[43] J.P. Becker, F. Van Bambeke, P.M. Tulkens, M. Prevost, Dynamics and structural changes induced by ATP binding in SAV1866, a bacterial ABC exporter, J. Phys. Chem. B 114 (2010) 15948-15957.

[44] G. Kaul, G. Pattan, MsbA ATP-binding cassette (ABC) transporter of $E$. coli: structure and possible flippase mechanism, Indian J. Biochem. Biophys. 48 (2011) 7-13.

[45] R.J. Dawson, K.P. Locher, Structure of the multidrug ABC transporter Sav 1866 from Staphylococcus aureus in complex with AMP-PNP, FEBS Lett. 581 (2007) 935-938.

[46] R. Doshi, H.W. van Veen, Substrate binding stabilizes a pre-translocation intermediate in the ATP-binding cassette transport protein MsbA, J. Biol. Chem. 288 (2013) $21638-21647$.

[47] S.G. Aller, J. Yu, A. Ward, Y. Weng, S. Chittaboina, R. Zhuo, P.M. Harrell, Y.T. Trinh, Q. Zhang, I.L. Urbatsch, G. Chang, Structure of P-glycoprotein reveals a molecular basis for poly-specific drug binding, Science 323 (2009) 1718-1722.

[48] M. Hohl, C. Briand, M.G. Grutter, M.A. Seeger, Crystal structure of a heterodimeric $\mathrm{ABC}$ transporter in its inward-facing conformation, Nat. Struct. Mol. Biol. 19 (2012) 395-402.

[49] M. Herget, G. Oancea, S. Schrodt, M. Karas, R. Tampe, R. Abele, Mechanism of substrate sensing and signal transmission within an ABC transporter: use of a Trojan horse strategy, J. Biol. Chem. 282 (2007) 3871-3880.

[50] G. Oancea, M.L. O'Mara, W.F. Bennett, D.P. Tieleman, R. Abele, R. Tampe, Structural arrangement of the transmission interface in the antigen ABC transport complex TAP, Proc. Natl. Acad. Sci. U. S. A. 106 (2009) 5551-5556.

[51] X. Guo, J. Li, T. Wang, Z. Liu, X. Chen, Y. Li, Z. Gu, X. Mao, W. Guan, Y. Li, A mutation in intracellular loop 4 affects the drug-efflux activity of the yeast multidrug resistance ABC transporter Pdr5p, PLoS One 7 (2012) e29520.

[52] R.J. Dawson, K. Hollenstein, K.P. Locher, Uptake or extrusion: crystal structures of full ABC transporters suggest a common mechanism, Mol. Microbiol. 65 (2007) 250-257.

[53] M.K. Al-Shawi, Catalytic and transport cycles of ABC exporters, Essays Biochem. 50 (2011) 63-83.

[54] K. Hollenstein, D.C. Frei, K.P. Locher, Structure of an ABC transporter in complex with its binding protein, Nature 446 (2007) 213-216.

[55] S. Gerber, M. Comellas-Bigler, B.A. Goetz, K.P. Locher, Structural basis of transinhibition in a molybdate/tungstate ABC transporter, Science 321 (2008) 246-250.

[56] E. Johnson, P.T. Nguyen, T.O. Yeates, D.C. Rees, Inward facing conformations of the MetNI methionine ABC transporter: implications for the mechanism of transinhibition, Protein Sci. 21 (2012) 84-96.

[57] M.L. Oldham, D. Khare, F.A. Quiocho, A.L. Davidson, J. Chen, Crystal structure of a catalytic intermediate of the maltose transporter, Nature 450 (2007) 515-521.
[58] A.L. Davidson, E. Dassa, C. Orelle, J. Chen, Structure, function, and evolution of bacterial ATP-binding cassette systems, Microbiol. Mol. Biol. Rev. MMBR 72 (2008) 317-364.

[59] E. Procko, M.L. O'Mara, W.F. Bennett, D.P. Tieleman, R. Gaudet, The mechanism of $A B C$ transporters: general lessons from structural and functional studies of an antigenic peptide transporter, FASEB J. 23 (2009) 1287-1302.

[60] K. Brillet, F. Ruffenach, H. Adams, L. Journet, V. Gasser, F. Hoegy, L. Guillon, M. Hannauer, A. Page, I.J. Schalk, An ABC transporter with two periplasmic binding proteins involved in iron acquisition in Pseudomonas aeruginosa, ACS Chem. Biol. 7 (2012) 2036-2045

[61] M. Mourez, M. Hofnung, E. Dassa, Subunit interactions in ABC transporters: a conserved sequence in hydrophobic membrane proteins of periplasmic permeases defines an important site of interaction with the ATPase subunits, EMBO J. 16 (1997) 3066-3077.

[62] P.C. Wen, E. Tajkhorshid, Conformational coupling of the nucleotide-binding and the transmembrane domains in ABC transporters, Biophys. J. 101 (2011) 680-690

[63] Smriti, S. Krishnamurthy, B.L. Dixit, C.M. Gupta, S. Milewski, R. Prasad, ABC transporters Cdr1p, Cdr2p and Cdr3p of a human pathogen Candida albicans are general phospholipid translocators, Yeast 19 (2002) 303-318.

[64] F. Quazi, S. Lenevich, R.S. Molday, ABCA4 is an N-retinylidenephosphatidylethanolamine and phosphatidylethanolamine importer, Nat. Commun. 3 (2012) 925.

[65] F. Quazi, R.S. Molday, Differential phospholipid substrates and directional transport by ATP binding cassette proteins ABCA1, ABCA7, and ABCA4 and disease-causing mutants, J. Biol. Chem. 288 (2013) 34414-34426.

[66] I. Balan, A.M. Alarco, M. Raymond, The Candida albicans CDR3 gene codes for an opaque-phase ABC transporter, J. Bacteriol. 179 (1997) 7210-7218.

[67] Y. Tsybovsky, R.S. Molday, K. Palczewski, The ATP-binding cassette transporter ABCA4: structural and functional properties and role in retinal disease, Adv. Exp. Med. Biol. 703 (2010) 105-125.

[68] Y. Kamimoto, K. Terasaka, M. Hamamoto, K. Takanashi, S. Fukuda, N. Shitan, A. Sugiyama, H. Suzuki, D. Shibata, B. Wang, S. Pollmann, M. Geisler, K. Yazaki, Arabidopsis ABCB21 is a facultative auxin importer/exporter regulated by cytoplasmic auxin concentration, Plant Cell Physiol. 53 (2012) 2090-2100.

[69] M. Kubes, H. Yang, G.L. Richter, Y. Cheng, E. Mlodzinska, X. Wang, J.J. Blakeslee, N. Carraro, J. Petrasek, E. Zazimalova, K. Hoyerova, W.A. Peer, A.S. Murphy, The Arabidopsis concentration-dependent influx/efflux transporter ABCB4 regulate cellular auxin levels in the root epidermis, Plant J. 69 (2012) 640-654.

[70] P.D. Eckford, F.J. Sharom, The reconstituted P-glycoprotein multidrug transporter is a flippase for glucosylceramide and other simple glycosphingolipids, Biochem. J. 389 (2005) 517-526

[71] D. Halter, S. Neumann, S.M. van Dijk, J. Wolthoorn, A.M. de Maziere, O.V. Vieira, P. Mattjus, J. Klumperman, G. van Meer, H. Sprong, Pre- and post-Golgi translocation of glucosylceramide in glycosphingolipid synthesis, J. Cell Biol. 179 (2007) 101-115.

[72] P.D. Eckford, F.J. Sharom, The reconstituted Escherichia coli MsbA protein displays lipid flippase activity, Biochem. J. 429 (2010) 195-203.

[73] J. Cui, A.L. Davidson, ABC solute importers in bacteria, Essays Biochem. 50 (2011) 85-99.

[74] C.F. Higgins, K.J. Linton, The ATP switch model for ABC transporters, Nat. Struct. Mol. Biol. 11 (2004) 918-926.

[75] M.E. Zoghbi, S. Krishnan, G.A. Altenberg, Dissociation of ATP-binding cassette nucleotide-binding domain dimers into monomers during the hydrolysis cycle, J. Biol. Chem. 287 (2012) 14994-15000.

[76] M.S. Jin, M.L. Oldham, Q. Zhang, J. Chen, Crystal structure of the multidrug transporter P-glycoprotein from Caenorhabditis elegans, Nature 490 (2012) 566-569.

[77] G. Gyimesi, S. Ramachandran, P. Kota, N.V. Dokholyan, B. Sarkadi, T. Hegedus, ATP hydrolysis at one of the two sites in $A B C$ transporters initiates transport related conformational transitions, Biochim. Biophys. Acta 1808 (2011) 2954-2964.

[78] A.E. Senior, M.K. al-Shawi, I.L. Urbatsch, The catalytic cycle of P-glycoprotein, FEBS Lett. 377 (1995) 285-289.

[79] P.M. Jones, A.M. George, Opening of the ADP-bound active site in the ABC transporter ATPase dimer: evidence for a constant contact, alternating sites model for the catalytic cycle, Proteins 75 (2009) 387-396

[80] P.M. Jones, A.M. George, Mechanism of the ABC transporter ATPase domains: catalytic models and the biochemical and biophysical record, Crit. Rev. Biochem. Mol. Biol. 48 (2013) 39-50.

[81] R.M. Krupka, Uncoupled active transport mechanisms accounting for low selectivity in multidrug carriers: P-glycoprotein and SMR antiporters, J. Membr. Biol. 172 (1999) 129-143.

[82] P. Seelheim, A. Wullner, H.J. Galla, Substrate translocation and stimulated ATP hydrolysis of human $\mathrm{ABC}$ transporter MRP3 show positive cooperativity and are half-coupled, Biophys. Chem. 171 (2013) 31-37.

[83] G. Tombline, A. Muharemagic, L.B. White, A.E. Senior, Involvement of the "occluded nucleotide conformation" of P-glycoprotein in the catalytic pathway, Biochemistry-Us 44 (2005) 12879-12886.

[84] E.O. Oloo, D.P. Tieleman, Conformational transitions induced by the binding of MgATP to the vitamin B12 ATP-binding cassette $(A B C)$ transporter BtuCD, J. Biol. Chem. 279 (2004) 45013-45019.

[85] P.M. Jones, A.M. George, Nucleotide-dependent allostery within the ABC transporter ATP-binding cassette: a computational study of the MJ0796 dimer, J. Biol. Chem. 282 (2007) 22793-22803.

[86] J.Y. Lee, I.L. Urbatsch, A.E. Senior, S. Wilkens, Nucleotide-induced structural changes in P-glycoprotein observed by electron microscopy, J. Biol. Chem. 283 (2008) 5769-5779.

[87] Z. Zhou, K.A. White, A. Polissi, C. Georgopoulos, C.R. Raetz, Function of Escherichia coli MsbA, an essential ABC family transporter, in lipid A and phospholipid biosynthesis, J. Biol. Chem. 273 (1998) 12466-12475. 
[88] W.T. Doerrler, M.C. Reedy, C.R. Raetz, An Escherichia coli mutant defective in lipid export, J. Biol. Chem. 276 (2001) 11461-11464.

[89] A. van Helvoort, A.J. Smith, H. Sprong, I. Fritzsche, A.H. Schinkel, P. Borst, G. van Meer, MDR1 P-glycoprotein is a lipid translocase of broad specificity, while MDR3 P-glycoprotein specifically translocates phosphatidylcholine, Cell 87 (1996) 507-517.

[90] Y. Romsicki, F.J. Sharom, Phospholipid flippase activity of the reconstituted P-glycoprotein multidrug transporter, Biochemistry-Us 40 (2001) 6937-6947.

[91] C. Vedhachalam, P.T. Duong, M. Nickel, D. Nguyen, P. Dhanasekaran, H. Saito, G.H. Rothblat, S. Lund-Katz, M.C. Phillips, Mechanism of ATP-binding cassette transporter A1-mediated cellular lipid efflux to apolipoprotein A-I and formation of high density lipoprotein particles, J. Biol. Chem. 282 (2007) 25123-25130.

[92] G. Bradley, V. Ling, P-glycoprotein, multidrug resistance and tumor progression, Cancer Metastasis Rev. 13 (1994) 223-233.

[93] A. Kodan, T. Yamaguchi, T. Nakatsu, K. Sakiyama, C.J. Hipolito, A. Fujioka, R Hirokane, K. Ikeguchi, B. Watanabe, J. Hiratake, Y. Kimura, H. Suga, K. Ueda, H Kato, Structural basis for gating mechanisms of a eukaryotic P-glycoprotein homolog, Proc. Natl. Acad. Sci. U. S. A. 111 (2014) 4049-4054.

[94] P. Zou, H.S. McHaourab, Alternating access of the putative substrate-binding chamber in the ABC transporter MsbA, J. Mol. Biol. 393 (2009) 574-585.

[95] A. Pohl, P.F. Devaux, A. Herrmann, Function of prokaryotic and eukaryotic ABC proteins in lipid transport, Biochim. Biophys. Acta 1733 (2005) 29-52.

[96] G. van Meer, D. Halter, H. Sprong, P. Somerharju, M.R. Egmond, ABC lipid transporters: extruders, flippases, or flopless activators? FEBS Lett. 580 (2006) 1171-1177.

[97] G. King, F.J. Sharom, Proteins that bind and move lipids: MsbA and NPC1, Crit. Rev. Biochem. Mol. Biol. 47 (2012) 75-95.

[98] K.B. Axelsen, M.G. Palmgren, Evolution of substrate specificities in the P-type ATPase superfamily, J. Mol. Evol. 46 (1998) 84-101.

[99] B.P. Pedersen, G. Ifrim, P. Liboriussen, K.B. Axelsen, M.G. Palmgren, P. Nissen, C Wiuf, C.N. Pedersen, Large scale identification and categorization of protein sequences using structured logistic regression, PLoS One 9 (2014) e85139.

[100] C. Toyoshima, M. Nakasako, H. Nomura, H. Ogawa, Crystal structure of the calcium pump of sarcoplasmic reticulum at 2.6 A resolution, Nature 405 (2000) 647-655.

[101] L. Baekgaard, A.T. Fuglsang, M.G. Palmgren, Regulation of plant plasma membrane $\mathrm{H}+-$ and $\mathrm{Ca}^{2+}$-ATPases by terminal domains, J. Bioenerg. Biomembr. 37 (2005) 369-374.

[102] K. Ekberg, M.G. Palmgren, B. Veierskov, M.J. Buch-Pedersen, A novel mechanism of P-type ATPase autoinhibition involving both termini of the protein, J. Biol. Chem 285 (2010) 7344-7350.

[103] M.G. Palmgren, P. Nissen, P-type ATPases, Annu. Rev. Biophys. 40 (2011) 243-266.

[104] E. Gomes, M.K. Jakobsen, K.B. Axelsen, M. Geisler, M.G. Palmgren, Chilling tolerance in Arabidopsis involves ALA1, a member of a new family of putative aminophospholipid translocases, Plant Cell 12 (2000) 2441-2454.

[105] M. Bublitz, H. Poulsen, J.P. Morth, P. Nissen, In and out of the cation pumps: P-type ATPase structure revisited, Curr. Opin. Struct. Biol. 20 (2010) 431-439.

[106] R.W. Albers, Biochemical aspects of active transport, Annu. Rev. Biochem. 36 (1967) 727-756.

[107] R.L. Post, S. Kume, T. Tobin, B. Orcutt, A.K. Sen, Flexibility of an active center in sodium-plus-potassium adenosine triphosphatase, J. Biol. Chem. 54 (1969) 306-326.

[108] M. Bublitz, J.P. Morth, P. Nissen, P-type ATPases at a glance, J. Cell Sci. 124 (2011) 2515-2519.

[109] K.B. Axelsen, M.G. Palmgren, Inventory of the superfamily of P-type ion pumps in Arabidopsis, Plant Physiol. 126 (2001) 696-706.

[110] B.P. Pedersen, M.J. Buch-Pedersen, J.P. Morth, M.G. Palmgren, P. Nissen, Crystal structure of the plasma membrane proton pump, Nature 450 (2007) 1111-1114.

[111] K. Saito, K. Fujimura-Kamada, N. Furuta, U. Kato, M. Umeda, K. Tanaka, Cdc50p, a protein required for polarized growth, associates with the Drs2p P-type ATPase implicated in phospholipid translocation in Saccharomyces cerevisiae, Mol. Biol. Cell 15 (2004) 3418-3432.

[112] L.R. Poulsen, R.L. Lopez-Marques, M.G. Palmgren, Flippases: still more questions than answers, Cell. Mol. Life Sci. 65 (2008) 3119-3125.

[113] L.R. Poulsen, R.L. Lopez-Marques, S.C. McDowell, J. Okkeri, D. Licht, A. Schulz, T. Pomorski, J.F. Harper, M.G. Palmgren, The Arabidopsis P4-ATPase ALA3 localizes to the golgi and requires a beta-subunit to function in lipid translocation and secretory vesicle formation, Plant Cell 20 (2008) 658-676.

[114] R.L. Lopez-Marques, L.R. Poulsen, S. Hanisch, K. Meffert, M.J. Buch-Pedersen, M.K. Jakobsen, T.G. Pomorski, M.G. Palmgren, Intracellular targeting signals and lipid specificity determinants of the ALA/ALIS P4-ATPase complex reside in the catalytic ALA alpha-subunit, Mol. Biol. Cell 21 (2010) 791-801.

[115] C.C. Paulusma, D.E. Folmer, K.S. Ho-Mok, D.R. de Waart, P.M. Hilarius, A.J Verhoeven, R.P. Oude Elferink, ATP8B1 requires an accessory protein for endoplasmic reticulum exit and plasma membrane lipid flippase activity, Hepatology 47 (2008) 268-278

[116] L.M. van der Velden, C.G. Wichers, A.E. van Breevoort, J.A. Coleman, R.S. Molday, R. Berger, L.W. Klomp, S.F. van de Graaf, Heteromeric interactions required for abundance and subcellular localization of human CDC50 proteins and class 1 P4ATPases, J. Biol. Chem. 285 (2010) 40088-40096.

[117] R.E. Dempski, T. Friedrich, E. Bamberg, The beta subunit of the $\mathrm{Na}+/ \mathrm{K}+-$ ATPase follows the conformational state of the holoenzyme, J. General Physiol. 125 (2005) 505-520.

[118] K. Geering, Functional roles of Na,K-ATPase subunits, Curr. Opin. Nephrol. Hypertens. 17 (2008) 526-532.

[119] P. Beguin, X. Wang, D. Firsov, A. Puoti, D. Claeys, J.D. Horisberger, K. Geering, The gamma subunit is a specific component of the Na,K-ATPase and modulates its transport function, EMBO J. 16 (1997) 4250-4260.
[120] S. Bryde, H. Hennrich, P.M. Verhulst, P.F. Devaux, G. Lenoir, J.C. Holthuis, CDC50 proteins are critical components of the human class-1 P4-ATPase transport machinery, J. Biol. Chem. 285 (2010) 40562-40572.

121] G. Lenoir, P. Williamson, C.F. Puts, J.C. Holthuis, Cdc50p plays a vital role in the ATPase reaction cycle of the putative aminophospholipid transporter Drs2p, J. Biol. Chem. 284 (2009) 17956-17967.

[122] R.D. Baldridge, T.R. Graham, Identification of residues defining phospholipid flippase substrate specificity of type IV P-type ATPases, Proc. Natl. Acad. Sci. U. S. A. 109 (2012) 290-298.

[123] A. Stone, P. Williamson, Outside of the box: recent news about phospholipid translocation by P4 ATPases, J. Chem. Biol. 5 (2012) 131-136.

[124] J.A. Coleman, A.L. Vestergaard, R.S. Molday, B. Vilsen, J.P. Andersen, Critical role of a transmembrane lysine in aminophospholipid transport by mammalian photoreceptor P4-ATPase ATP8A2, Proc. Natl. Acad. Sci. U. S. A. 109 (2012) 1449-1454.

[125] R.D. Baldridge, T.R. Graham, Two-gate mechanism for phospholipid selection and transport by type IV P-type ATPases, Proc. Natl. Acad. Sci. U. S. A. 110 (2013) 358-367.

[126] A.L. Vestergaard, J.A. Coleman, T. Lemmin, S.A. Mikkelsen, L.L. Molday, B. Vilsen, R.S Molday, M. Dal Peraro, J.P. Andersen, Critical roles of isoleucine-364 and adjacent residues in a hydrophobic gate control of phospholipid transport by the mammalian P4-ATPase ATP8A2, Proc. Natl. Acad. Sci. U. S. A. 111 (2014) E1334-E1343.

[127] R.D. Baldridge, P. Xu, T.R. Graham, Type IV P-type ATPases distinguish monoversus diacyl phosphatidylserine using a cytofacial exit gate in the membrane domain, J. Biol. Chem. 288 (2013) 19516-19527.

[128] K. Obara, N. Miyashita, C. Xu, I. Toyoshima, Y. Sugita, G. Inesi, C. Toyoshima, Structural role of countertransport revealed in $\mathrm{Ca}^{2+}$ pump crystal structure in the absence of $\mathrm{Ca}^{2+}$, Proc. Natl. Acad. Sci. U. S. A. 102 (2005) 14489-14496.

[129] C. Olesen, M. Picard, A.M. Winther, C. Gyrup, J.P. Morth, C. Oxvig, J.V. Moller, P. Nissen, The structural basis of calcium transport by the calcium pump, Nature 450 (2007) 1036-1042.

[130] Y. Taguchi, A. Yoshida, Y. Takada, T. Komano, K. Ueda, Anti-cancer drugs and glutathione stimulate vanadate-induced trapping of nucleotide in multidrug resistanceassociated protein (MRP), FEBS Lett. 401 (1997) 11-14.

[131] A. Wach, P. Graber, The plasma membrane $\mathrm{H}^{+}$-ATPase from yeast. Effects of $\mathrm{pH}$, vanadate and erythrosine B on ATP hydrolysis and ATP binding, Eur. J. Biochem. 201 (1991) 91-97.

[132] S. Sharma, A.L. Davidson, Vanadate-induced trapping of nucleotides by purified maltose transport complex requires ATP hydrolysis, J. Bacteriol. 182 (2000) 6570-6576.

[133] C. Olesen, T.L. Sorensen, R.C. Nielsen, J.V. Moller, P. Nissen, Dephosphorylation of the calcium pump coupled to counterion occlusion, Science 306 (2004) 2251-2255.

134] A. Jacquot, C. Montigny, H. Hennrich, R. Barry, M. le Maire, C. Jaxel, J. Holthuis, P. Champeil, G. Lenoir, Phosphatidylserine stimulation of Drs2p.Cdc50p lipid translocase dephosphorylation is controlled by phosphatidylinositol-4phosphate, J. Biol. Chem. 287 (2012) 13249-13261.

[135] A. Groen, M.R. Romero, C. Kunne, S.J. Hoosdally, P.H. Dixon, C. Wooding, C. Williamson, J. Seppen, K. Van den Oever, K.S. Mok, C.C. Paulusma, K.J. Linton, R.P. Oude Elferink, Complementary functions of the flippase ATP8B1 and the floppase ABCB4 in maintaining canalicular membrane integrity, Gastroenterology 141 (2011) 1927-1937

[136] M. Caffrey, Crystallizing membrane proteins for structure determination: use of lipidic mesophases, Annu. Rev. Biophys. 38 (2009) 29-51.

[137] W.T. Doerrler, C.R. Raetz, ATPase activity of the MsbA lipid flippase of Escherichia coli, J. Biol. Chem. 277 (2002) 36697-36705.

[138] W.T. Doerrler, H.S. Gibbons, C.R. Raetz, MsbA-dependent translocation of lipids across the inner membrane of Escherichia coli, J. Biol. Chem. 279 (2004) 45102-45109.

[139] P.D. Eckford, F.J. Sharom, Functional characterization of Escherichia coli MsbA: interaction with nucleotides and substrates, J. Biol. Chem. 283 (2008) 12840-12850.

[140] C. Alaimo, I. Catrein, L. Morf, C.L. Marolda, N. Callewaert, M.A. Valvano, M.F. Feldman, M. Aebi, Two distinct but interchangeable mechanisms for flipping of lipid-linked oligosaccharides, EMBO J. 25 (2006) 967-976.

[141] A. Margolles, M. Putman, H.W. van Veen, W.N. Konings, The purified and functionally reconstituted multidrug transporter LmrA of Lactococcus lactis mediates the transbilayer movement of specific fluorescent phospholipids, Biochemistry-Us 38 (1999) 16298-16306.

[142] G. Reuter, T. Janvilisri, H. Venter, S. Shahi, L. Balakrishnan, H.W. van Veen, The ATP binding cassette multidrug transporter LmrA and lipid transporter MsbA have overlapping substrate specificities, J. Biol. Chem. 278 (2003) 35193-35198.

[143] A. Decottignies, A.M. Grant, J.W. Nichols, H. de Wet, D.B. McIntosh, A. Goffeau, ATPase and multidrug transport activities of the overexpressed yeast $A B C$ protein Yor1p, J. Biol. Chem. 273 (1998) 12612-12622.

[144] T. Pomorski, R. Lombardi, H. Riezman, P.F. Devaux, G. van Meer, J.C. Holthuis, Drs2p-related P-type ATPases Dnf1p and Dnf2p are required for phospholipid translocation across the yeast plasma membrane and serve a role in endocytosis, Mol. Biol. Cell 14 (2003) 1240-1254.

[145] K. Gulshan, W.S. Moye-Rowley, Vacuolar import of phosphatidylcholine requires the ATP-binding cassette transporter Ybt1, Traffic 12 (2011) 1257-1268.

[146] S. Dogra, S. Krishnamurthy, V. Gupta, B.L. Dixit, C.M. Gupta, D. Sanglard, R. Prasad, Asymmetric distribution of phosphatidylethanolamine in C. albicans: possible mediation by CDR1, a multidrug transporter belonging to ATP binding cassette (ABC) superfamily, Yeast 15 (1999) 111-121.

[147] S. Shukla, V. Rai, P. Saini, D. Banerjee, A.K. Menon, R. Prasad, Candida drug resistance protein 1, a major multidrug ATP binding cassette transporter of Candida albicans, translocates fluorescent phospholipids in a reconstituted system, Biochemistry-Us 46 (2007) 12081-12090. 
[148] A. Parodi-Talice, J.M. Araujo, C. Torres, J.M. Perez-Victoria, F. Gamarro, S. Castanys, The overexpression of a new $\mathrm{ABC}$ transporter in Leishmania is related to phospholipid trafficking and reduced infectivity, Biochim. Biophys. Acta 1612 (2003) 195-207.

[149] J.M. Araujo-Santos, A. Parodi-Talice, S. Castanys, F. Gamarro, The overexpression of an intracellular ABCA-like transporter alters phospholipid trafficking in Leishmania, Biochem. Biophys. Res. Commun. 330 (2005) 349-355.

[150] J.M. Perez-Victoria, F.J. Perez-Victoria, A. Parodi-Talice, I.A. Jimenez, A.G. Ravelo, S. Castanys, F. Gamarro, Alkyl-lysophospholipid resistance in multidrug-resistant Leishmania tropica and chemosensitization by a novel P-glycoprotein-like transporter modulator, Antimicrob. Agents Chemother. 45 (2001) 2468-2474.

[151] J. Campos-Salinas, D. Leon-Guerrero, E. Gonzalez-Rey, M. Delgado, S. Castanys, J.M. Perez-Victoria, F. Gamarro, LABCG2, a new ABC transporter implicated in phosphatidylserine exposure, is involved in the infectivity and pathogenicity of Leishmania, PLoS Negl. Trop. Dis. 7 (2013) e2179.

[152] E. Castanys-Munoz, N. Alder-Baerens, T. Pomorski, F. Gamarro, S. Castanys, A novel ATP-binding cassette transporter from Leishmania is involved in transport of phosphatidylcholine analogues and resistance to alkyl-phospholipids, Mol. Microbiol. 64 (2007) 1141-1153.

[153] E. Castanys-Munoz, J.M. Perez-Victoria, F. Gamarro, S. Castanys, Characterization of an ABCG-like transporter from the protozoan parasite Leishmania with a role in drug resistance and transbilayer lipid movement, Antimicrob. Agents Chemother. 52 (2008) 3573-3579.

[154] Y. Matsumura, N. Ban, N. Inagaki, Aberrant catalytic cycle and impaired lipid transport into intracellular vesicles in ABCA3 mutants associated with nonfatal pediatric interstitial lung disease, Am. J. Physiol. Lung Cell. Mol. Physiol. 295 (2008) 698-707.

[155] Y. Matsumura, H. Sakai, M. Sasaki, N. Ban, N. Inagaki, ABCA3-mediated cholinephospholipids uptake into intracellular vesicles in A549 cells, FEBS Lett. 581 (2007) 3139-3144.

[156] N. Cheong, M. Madesh, L.W. Gonzales, M. Zhao, K. Yu, P.L. Ballard, H. Shuman, Functional and trafficking defects in ATP binding cassette A3 mutants associated with respiratory distress syndrome, J. Biol. Chem. 281 (2006) 9791-9800.

[157] S. Beharry, M. Zhong, R.S. Molday, N-retinylidene-phosphatidylethanolamine is the preferred retinoid substrate for the photoreceptor-specific ABC transporter ABCA4 (ABCR), J. Biol. Chem. 279 (2004) 53972-53979.

[158] N. Wang, D. Lan, M. Gerbod-Giannone, P. Linsel-Nitschke, A.W. Jehle, W. Chen, L.O. Martinez, A.R. Tall, ATP-binding cassette transporter A7 (ABCA7) binds apolipoprotein A-I and mediates cellular phospholipid but not cholesterol efflux, J. Biol. Chem. 278 (2003) 42906-42912.

[159] S. Abe-Dohmae, Y. Ikeda, M. Matsuo, M. Hayashi, K. Okuhira, K. Ueda, S. Yokoyama, Human ABCA7 supports apolipoprotein-mediated release of cellular cholesterol and phospholipid to generate high density lipoprotein, J. Biol. Chem. 279 (2004) 604-611.

[160] P. Linsel-Nitschke, A.W. Jehle, J. Shan, G. Cao, D. Bacic, D. Lan, N. Wang, A.R. Tall, Potential role of ABCA7 in cellular lipid efflux to apoA-I, J. Lipid Res. 46 (2005) $86-92$.

[161] I. Bosch, K. Dunussi-Joannopoulos, R.L. Wu, S.T. Furlong, J. Croop, Phosphatidylcholine and phosphatidylethanolamine behave as substrates of the human MDR1 P-glycoprotein, Biochemistry-Us 36 (1997) 5685-5694.

[162] A.G. Abulrob, M. Gumbleton, Transport of phosphatidylcholine in MDR3-negative epithelial cell lines via drug-induced MDR1 P-glycoprotein, Biochem. Biophys. Res. Commun. 262 (1999) 121-126.

[163] S. Ernest, E. Bello-Reuss, Secretion of platelet-activating factor is mediated by MDR1 P-glycoprotein in cultured human mesangial cells, J. Am. Soc. Nephrol. JASN 10 (1999) 2306-2313.

[164] A. Pohl, H. Lage, P. Muller, T. Pomorski, A. Herrmann, Transport of phosphatidylserine via MDR1 (multidrug resistance 1)P-glycoprotein in a human gastric carcinoma cell line, Biochem. J. 365 (2002) 259-268.

[165] J.J. Smit, A.H. Schinkel, R.P. Oude Elferink, A.K. Groen, E. Wagenaar, L. van Deemter, C.A. Mol, R. Ottenhoff, N.M. van der Lugt, M.A. van Roon, et al., Homozygous disruption of the murine mdr2 P-glycoprotein gene leads to a complete absence of phospholipid from bile and to liver disease, Cell 75 (1993) 451-462.

[166] A.J. Smith, J.L. Timmermans-Hereijgers, B. Roelofsen, K.W. Wirtz, W.J. van Blitterswijk, J.J. Smit, A.H. Schinkel, P. Borst, The human MDR3 P-glycoprotein promotes translocation of phosphatidylcholine through the plasma membrane of fibroblasts from transgenic mice, FEBS Lett. 354 (1994) 263-266.

[167] S. Ruetz, P. Gros, Phosphatidylcholine translocase: a physiological role for the mdr2 gene, Cell 77 (1994) 1071-1081.

[168] R.J. Raggers, A. van Helvoort, R. Evers, G. van Meer, The human multidrug resistance protein MRP1 translocates sphingolipid analogs across the plasma membrane, J. Cell Sci. 112 (Pt 3) (1999) 415-422.

[169] Z. Huang, X. Chang, J.R. Riordan, Y. Huang, Fluorescent modified phosphatidylcholine floppase activity of reconstituted multidrug resistance-associated protein MRP1, Biochim. Biophys. Acta 1660 (2004) 155-163.

[170] A. Sohnius, D. Kamp, C.W. Haest, ATP and GSH dependence of MRP1-mediated outward translocation of phospholipid analogs in the human erythrocyte membrane, Mol. Membr. Biol. 20 (2003) 299-305.

[171] D. Kamp, C.W. Haest, Evidence for a role of the multidrug resistance protein (MRP) in the outward translocation of NBD-phospholipids in the erythrocyte membrane, Biochim. Biophys. Acta 1372 (1998) 91-101.

[172] W.R. Riekhof, D.R. Voelker, Uptake and utilization of lyso-phosphatidylethanolamine by Saccharomyces cerevisiae, J. Biol. Chem. 281 (2006) 36588-36596.

[173] W.R. Riekhof, J. Wu, M.A. Gijon, S. Zarini, R.C. Murphy, D.R. Voelker, Lysophosphatidylcholine metabolism in Saccharomyces cerevisiae: the role of P-type
ATPases in transport and a broad specificity acyltransferase in acylation, J. Biol. Chem. 282 (2007) 36853-36861.

[174] P. Xu, R.D. Baldridge, R.J. Chi, C.G. Burd, T.R. Graham, Phosphatidylserine flipping enhances membrane curvature and negative charge required for vesicular transport, J. Cell Biol. 202 (2013) 875-886.

[175] N. Alder-Baerens, Q. Lisman, L. Luong, T. Pomorski, J.C. Holthuis, Loss of P4 ATPases Drs2p and Dnf3p disrupts aminophospholipid transport and asymmetry in yeast post-Golgi secretory vesicles, Mol. Biol. Cell 17 (2006) 1632-1642.

[176] X. Zhou, T.R. Graham, Reconstitution of phospholipid translocase activity with purified Drs2p, a type-IV P-type ATPase from budding yeast, Proc. Natl. Acad. Sci. U. S. A. 106 (2009) 16586-16591.

[177] F.J. Perez-Victoria, M.P. Sanchez-Canete, S. Castanys, F. Gamarro, Phospholipid translocation and miltefosine potency require both $L$. donovani miltefosine transporter and the new protein LdRos3 in Leishmania parasites, J. Biol. Chem. 281 (2006) 23766-23775.

[178] A. Weingartner, B. Drobot, A. Herrmann, M.P. Sanchez-Canete, F. Gamarro, S. Castanys, T. Gunther Pomorski, Disruption of the lipid-transporting LdMTLdRos3 complex in Leishmania donovani affects membrane lipid asymmetry but not host cell invasion, PLoS One 5 (2010) e12443.

[179] B. Chen, Y. Jiang, S. Zeng, J. Yan, X. Li, Y. Zhang, W. Zou, X. Wang, Endocytic sorting and recycling require membrane phosphatidylserine asymmetry maintained by TAT-1/CHAT-1, PLoS Genet. 6 (2010) e1001235.

[180] M. Darland-Ransom, X. Wang, C.L. Sun, J. Mapes, K. Gengyo-Ando, S. Mitani, D. Xue, Role of $C$. elegans TAT-1 protein in maintaining plasma membrane phosphatidylserine asymmetry, Science 320 (2008) 528-531.

[181] A.M. Wehman, C. Poggioli, P. Schweinsberg, B.D. Grant, J. Nance, The P4-ATPase TAT-5 inhibits the budding of extracellular vesicles in C. elegans embryos, Curr. Biol. CB 21 (2011) (1951-1959).

[182] R.L. Lopez-Marques, L.R. Poulsen, M.G. Palmgren, A putative plant aminophospholipid flippase, the Arabidopsis P4 ATPase ALA1, localizes to the plasma membrane following association with a beta-subunit, PLoS One 7 (2012) e33042.

[183] J.K. Paterson, K. Renkema, L. Burden, M.S. Halleck, R.A. Schlegel, P. Williamson, D.L Daleke, Lipid specific activation of the murine P4-ATPase Atp8a1 (ATPase II), Biochemistry-Us 45 (2006) 5367-5376.

[184] E. Soupene, D.U. Kemaladewi, F.A. Kuypers, ATP8A1 activity and phosphatidylserine transbilayer movement, J. Receptor Ligand Channel Res. 1 (2008) 1-10.

[185] U. Kato, H. Inadome, M. Yamamoto, K. Emoto, T. Kobayashi, M. Umeda, Role for phospholipid flippase complex of ATP8A1 and CDC50A proteins in cell migration, J. Biol. Chem. 288 (2013) 4922-4934.

[186] K. Levano, V. Punia, M. Raghunath, P.R. Debata, G.M. Curcio, A. Mogha, S. Purkayastha, D. McCloskey, J. Fata, P. Banerjee, Atp8a1 deficiency is associated with phosphatidylserine externalization in hippocampus and delayed hippocampus-dependent learning, J. Neurochem. 120 (2012) 302-313.

[187] J.A. Coleman, M.C. Kwok, R.S. Molday, Localization, purification, and functional reconstitution of the P4-ATPase Atp8a2, a phosphatidylserine flippase in photoreceptor disc membranes, J. Biol. Chem. 284 (2009) 32670-32679.

[188] J.A. Coleman, R.S. Molday, Critical role of the beta-subunit CDC50A in the stable expression, assembly, subcellular localization, and lipid transport activity of the P4-ATPase ATP8A2, J. Biol. Chem. 286 (2011) 17205-17216.

[189] P. Ujhazy, D. Ortiz, S. Misra, S. Li, J. Moseley, H. Jones, I.M. Arias, Familia intrahepatic cholestasis 1: studies of localization and function, Hepatology 34 (2001) 768-775.

[190] F. Munoz-Martinez, C. Torres, S. Castanys, F. Gamarro, CDC50A plays a key role in the uptake of the anticancer drug perifosine in human carcinoma cells, Biochem. Pharmacol. 80 (2010) 793-800.

[191] L. Wang, C. Beserra, D.L. Garbers, A novel aminophospholipid transporter exclusively expressed in spermatozoa is required for membrane lipid asymmetry and normal fertilization, Dev. Biol. 267 (2004) 203-215.

[192] M. Yabas, C.E. Teh, S. Frankenreiter, D. Lal, C.M. Roots, B. Whittle, D.T. Andrews, Y. Zhang, N.C. Teoh, J. Sprent, L.E. Tze, E.M. Kucharska, J. Kofler, G.C. Farell, S. Broer C.C. Goodnow, A. Enders, ATP11C is critical for the internalization of phosphatidylserine and differentiation of B lymphocytes, Nat. Immunol. 12 (2011) 441-449.

[193] A.B.. Ward, P. Szewczyk, V. Grimard, C.W. Lee, L. Martinez, R. Doshi, A. Caya, M. Villaluz, E. Pardon, C. Cregger, D.J. Swartz, P.G. Falson, I.L. Urbatsch, C. Govaerts, J. Steyaert, G. Chang, Structures of P-glycoprotein reveal its conformational flexibility and an epitope on the nucleotide-binding domain, Proc. Natl. Acad. Sci. U. S. A. 110 (2013) 13386-13391.

[194] J. Li, K.F. Jaimes, S.G. Aller, Refined structures of mouse P-glycoprotein, Protein Sci. 23 (2014) 34-46.

[195] L. Yatime, M. Laursen, J.P. Morth, M. Esmann, P. Nissen, N.U. Fedosova, Structural insights into the high affinity binding of cardiotonic steroids to the $\mathrm{Na}+$, K+-ATPase, J. Struct. Biol. 174 (2011) 296-306.

[196] J.P. Morth, B.P. Pedersen, M.S. Toustrup-Jensen, T.L. Sorensen, J. Petersen, J.P. Andersen, B. Vilsen, P. Nissen, Crystal structure of the sodium-potassium pump Nature 450 (2007) 1043-1049.

[197] H. Ogawa, T. Shinoda, F. Cornelius, C. Toyoshima, Crystal structure of the sodiumpotassium pump ( $\mathrm{Na}+, \mathrm{K}+$-ATPase) with bound potassium and ouabain, Proc. Natl. Acad. Sci. U. S. A. 106 (2009) 13742-13747.

[198] M. Nyblom, H. Poulsen, P. Gourdon, L. Reinhard, M. Andersson, E. Lindahl, N. Fedosova, P. Nissen, Crystal structure of $\mathrm{Na}^{+}, \mathrm{K}^{+}$-ATPase in the $\mathrm{Na}^{+}$-bound state Science 342 (2013) 123-127.

[199] T. Shinoda, H. Ogawa, F. Cornelius, C. Toyoshima, Crystal structure of the sodiumpotassium pump at 2.4 A resolution, Nature 459 (2009) 446-450. 
[200] R. Kanai, H. Ogawa, B. Vilsen, F. Cornelius, C. Toyoshima, Crystal structure of a Na+bound $\mathrm{Na}+, \mathrm{K}+-$-ATPase preceding the E1P state, Nature 502 (2013) 201-206.

[201] P. Gourdon, X.Y. Liu, T. Skjorringe, J.P. Morth, L.B. Moller, B.P. Pedersen, P. Nissen, Crystal structure of a copper-transporting PIB-type ATPase, Nature 475 (2011) 59-64.

[202] M. Andersson, D. Mattle, O. Sitsel, T. Klymchuk, A.M. Nielsen, L.B. Moller, S.H. White, P. Nissen, P. Gourdon, Copper-transporting P-type ATPases use a unique ion-release pathway, Nat. Struct. Mol. Biol. 21 (2014) 43-48.

[203] A.M. Winther, M. Bublitz, J.L. Karlsen, J.V. Moller, J.B. Hansen, P. Nissen, M.J. BuchPedersen, The sarcolipin-bound calcium pump stabilizes calcium sites exposed to the cytoplasm, Nature 495 (2013) 265-269.

[204] C. Toyoshima, S. Iwasawa, H. Ogawa, A. Hirata, J. Tsueda, G. Inesi, Crystal structures of the calcium pump and sarcolipin in the $\mathrm{Mg}^{2+}$-bound $\mathrm{E} 1$ state, Nature 495 (2013) 260-264.

[205] R. Sacchetto, I. Bertipaglia, S. Giannetti, L. Cendron, F. Mascarello, E. Damiani, E. Carafoli, G. Zanotti, Crystal structure of sarcoplasmic reticulum $\mathrm{Ca}^{2+}$-ATPase (SERCA) from bovine muscle, J. Struct. Biol. 178 (2012) 38-44.

[206] A.M. Jensen, T.L. Sorensen, C. Olesen, J.V. Moller, P. Nissen, Modulatory and catalytic modes of ATP binding by the calcium pump, EMBO J. 25 (2006) 2305-2314.

[207] C. Toyoshima, H. Nomura, T. Tsuda, Lumenal gating mechanism revealed in calcium pump crystal structures with phosphate analogues, Nature 432 (2004) 361-368.

[208] C. Toyoshima, T. Mizutani, Crystal structure of the calcium pump with a bound ATP analogue, Nature 430 (2004) 529-535.

[209] T.L. Sorensen, J.V. Moller, P. Nissen, Phosphoryl transfer and calcium ion occlusion in the calcium pump, Science 304 (2004) 1672-1675.

[210] M. Bublitz, M. Musgaard, H. Poulsen, L. Thogersen, C. Olesen, B. Schiott, J.P. Morth, J.V. Moller, P. Nissen, Ion pathways in the sarcoplasmic reticulum $\mathrm{Ca}^{2+}$-ATPase, J. Biol. Chem. 288 (2013) 10759-10765.

[211] C. Toyoshima, S. Yonekura, J. Tsueda, S. Iwasawa, Trinitrophenyl derivatives bind differently from parent adenine nucleotides to $\mathrm{Ca}^{2+}$-ATPase in the absence of $\mathrm{Ca}^{2+}$, Proc. Natl. Acad. Sci. U. S. A. 108 (2011) 1833-1838.
[212] E. S. Paulsen, J Villadsen, E Tenori, H. Liu, D.F. Bonde, M.A. Lie, M. Bublitz, C. Olesen, H.E. Autzen, I. Dach, P. Sehgal, P. Nissen, J.V. Moller, B. Schiott, S.B. Christensen, Water-mediated interactions influence the binding of thapsigargin to sarco/ endoplasmic reticulum calcium adenosinetriphosphatase, J. Med. Chem. 56 (2013) 3609-3619.

[213] M. Laursen, M. Bublitz, K. Moncoq, C. Olesen, J.V. Moller, H.S. Young, P. Nissen, J.P. Morth, Cyclopiazonic acid is complexed to a divalent metal ion when bound to the sarcoplasmic reticulum $\mathrm{Ca}^{2+}$-ATPase, J. Biol. Chem. 284 (2009) 13513-13518.

[214] K. Moncoq, C.A. Trieber, H.S. Young, The molecular basis for cyclopiazonic acid inhibition of the sarcoplasmic reticulum calcium pump, J. Biol. Chem. 282 (2007) 9748-9757.

[215] B.L. Akin, T.D. Hurley, Z. Chen, L.R. Jones, The structural basis for phospholamban inhibition of the calcium pump in sarcoplasmic reticulum, J. Biol. Chem. 288 (2013) 30181-30191.

[216] Y. Sonntag, M. Musgaard, C. Olesen, B. Schiott, J.V. Moller, P. Nissen, L. Thogersen, Mutual adaptation of a membrane protein and its lipid bilayer during conformational changes, Nat. Commun. 2 (2011) 1-7.

[217] A.M. Winther, H. Liu, Y. Sonntag, C. Olesen, M. le Maire, H. Soehoel, C.E. Olsen, S.B. Christensen, P. Nissen, J.V. Moller, Critical roles of hydrophobicity and orientation of side chains for inactivation of sarcoplasmic reticulum $\mathrm{Ca}^{2+}$-ATPase with thapsigargin and thapsigargin analogs, J. Biol. Chem. 285 (2010) 28883-28892.

[218] M. Takahashi, Y. Kondou, C. Toyoshima, Interdomain communication in calcium pump as revealed in the crystal structures with transmembrane inhibitors, Proc. Natl. Acad. Sci. U. S. A. 104 (2007) 5800-5805.

[219] H. Sohoel, A.M. Jensen, J.V. Moller, P. Nissen, S.R. Denmeade, J.T. Isaacs, C.E. Olsen, S.B. Christensen, Natural products as starting materials for development of secondgeneration SERCA inhibitors targeted towards prostate cancer cells, Bioorg. Med. Chem. 14 (2006) 2810-2815.

[220] C. Toyoshima, H. Nomura, Structural changes in the calcium pump accompanying the dissociation of calcium, Nature 418 (2002) 605-611 